\title{
Fully Eulerian finite element approximation of a fluid-structure interaction problem in cardiac cells
}

\author{
A. Laadhari ${ }^{1}$, R. Ruiz-Baier ${ }^{1, * \dagger}$ and A. Quarteroni ${ }^{1,2}$ \\ ${ }^{1}$ CMCS-MATHICSE-SB, École Polytechnique Fédérale de Lausanne, CH-1015 Lausanne, Switzerland \\ ${ }^{2}$ MOX - Modellistica e Calcolo Scientifico, Dipartimento di Matematica, Politecnico di Milano, 20133 Milano, Italy
}

\begin{abstract}
SUMMARY
We propose in this paper an Eulerian finite element approximation of a coupled chemical fluid-structure interaction problem arising in the study of mesoscopic cardiac biomechanics. We simulate the active response of a myocardial cell (here considered as an anisotropic, hyperelastic, and incompressible material), the propagation of calcium concentrations inside it, and the presence of a surrounding Newtonian fluid. An active strain approach is employed to account for the mechanical activation, and the deformation of the cell membrane is captured using a level set strategy. We address in detail the main features of the proposed method, and we report several numerical experiments aimed at model validation. Copyright $\odot 2013$ John Wiley \& Sons, Ltd.
\end{abstract}

Received 6 November 2012; Revised 26 July 2013; Accepted 10 September 2013

KEY WORDS: level set method; finite element approximation; fluid-structure interaction; orthotropic hyperelastic model; active mechanics; cardiac cells

\section{INTRODUCTION}

We are interested in the numerical simulation of a coupled system modeling the interplay of the deformation of an isolated myocardial cell and the microscopic chemical reactions occurring inside the cell that generate active tension. This phenomenon has a considerable importance in several biomedical applications [1], such as tissue regeneration, resynchronization therapies, and arrhythmogenesis. Even if there is an increasing interest in such applications, also from the viewpoint of scientific computing and numerical methods, most of the contributions focus on the study of the excitation-contraction coupling in the heart at the tissue and organ levels (see, e.g., the review articles [2-4] and the references therein). The numerical aspects concerning the electro-mechanical and electro-chemical effects taking place at the cell and sub-cell levels have been much less regarded, and available studies focus mainly on zero-dimensional models for ion kinetics. Nowadays, clinical trials are able to provide detailed experimental observations of the strong interaction of these complex mechanisms allowing validation of the proposed models and methods. For instance, there exist qualitative and quantitative clinical evidence of the hyperelastic anisotropic behavior of cardiac myocytes [5] and of the specific influence of extracellular calcium concentrations on the contraction of single cells [6]. The most basic experimental setting consists in taking specimens (a single or a small group of cells) immersed in a blood-like fluid solution and putting them under strain while stresses and related measures are obtained via mechanical transducers [1,7].

In this work, we model the contractility of a cardiomyocyte surrounded by a Newtonian fluid and the mechano-chemical interactions between calcium species and active contraction generation.

*Correspondence to: R. Ruiz-Baier, Present address: CRET-FGSE, University of Lausanne, CH-1015 Lausanne, Switzerland.

†E-mail: ricardo.ruizbaier@unil.ch 
It is well known that a total Lagrangian setting for the underlying problem (where flow equations and ionic models must be rewritten in the reference configuration) may generate instabilities, volumetric locking phenomena, and non-admissible displacements. In the so-called ALE formulation, the fluid problem can be treated under either an Eulerian or Lagrangian method, whereas the solid motion is described via a Lagrangian approach $([8,9])$, and kinematic and dynamic coupling conditions can be embedded into trial spaces and set weakly. This results particularly useful in the case of small deformations; however, some difficulties can arise from the dependence of the solution on the domain geometry. Moreover, interface-tracking approaches may exhibit problems related to large structural deformations, free movement of the structure within a flow domain, and contact of the structure with the domain boundary or with neighboring structures or selfcontact configurations. Even if large deflections in the ALE framework employing full remeshing have been already addressed (see, e.g., [10] and the bibliography therein), several problems are still present, especially in three-dimensional cases where the computational burden is considerable. In the immersed boundary method [11], the interaction equations require a smooth approximation of the Dirac measure to ensure the communication between Eulerian and Lagrangian quantities, which is achieved by rewriting solid elastic forces into the fluid domain and the velocity field inducing the structure dynamics. The method involves a fixed mesh for the fluid domain and a free moving nonconforming mesh for the structure domain. The Lagrangian mesh is typically required to be approximately twice as fine as the background Eulerian grid to avoid leaks at fluid-structure interfaces, and the whole domain has to be re-meshed as the solid moves, resulting in additional computational cost. Moreover, in some cases, the coordinate transformation needed at each time step affects volume conservation during velocity interpolation [12]. Under a fully Eulerian description, one considers both fluid and structure as a single continuum model. This strategy avoids some difficulties related to, for example, mesh distortion; however, additional equations (typically transport of either displacements, stresses, or deformation gradients) need to be solved with a high accuracy to correctly capture the evolution of the deformation map.

Evidently, the choice for the description of motion will not only modify the model itself but will also have a strong influence on the numerical implementation. We formulate our coupled problem in a fully Eulerian framework, also motivated by the following: (1) the advantage that boundary conditions for the soft tissue can be naturally described in terms of the differences of densities and viscosities between the solid and the surrounding fluid (we introduce an implicit function that handles the fluid-structure interface and drives the switch between the fluid and solid stresses) and (2) a fully Eulerian approach permits to treat large deformations and contact effects without the need of remeshing techniques (see, e.g., [13-15]). Our work follows these lines, but it differs in that we employ a unique mesh and the same solver everywhere, and the coupling of stresses and fluid-structure interface conditions are incorporated by a level set method. In general, interface capturing can be pursued with either volume-of-fluid methods [16, 17], phase-field [18, 19], or level set techniques [20,21]. We follow [22] and implicitly capture the boundary of the elastic body as the zero iso-surface of a level set function with a nearly unit gradient. Our development has been further motivated by [23], where the approach of [22] is employed to simulate calcium-induced cardiomyocyte contraction. However, here, we incorporate several variants on the method and in the model itself. First, our method is entirely based on a finite element discretization on unstructured meshes, whereas the numerical examples in $[22,23]$ are obtained with finite differences. In addition, we employ a modified level set approach based on the imposition of local mass preservation via a Lagrange multiplier (see, e.g., [24,25]). By approximating a Dirac measure as in the immerse boundary method, we consider an active strain description of the activation mechanism based on the assumption of a multiplicative decomposition of the deformation gradient [26,27]. Finally, we adapt a phenomenological model for the calcium-driven mechanical activation following [28]. From the viewpoint of theoretical mechanics, the active strain approach is consistent in the sense that the modified energy functionals maintain the same stability properties of the passive hyperelastic material (as, e.g., polyconvexity), which is not generally true in so-called active stress approaches.

The paper is organized as follows. In Section 2, we introduce some required notation and provide a consistent mathematical setting for the formulation of the mechano-chemical model. Section 3 presents a description of the FEM and a discussion on some discretization choices. A set of 
numerical examples illustrating the main features of the model and the accuracy of the numerical method are listed in Section 4, and we close with some comments in Section 5. Details on the linearization procedures are provided in the Appendix.

\section{MATHEMATICAL MODEL FOR CELL ACTIVE CONTRACTION}

Let $\boldsymbol{x}$ represents the current position of a material particle of the domain $\Omega=\Omega_{t}$ occupied by the cardiomyocyte, which corresponded to the position $\xi$ in the reference configuration $\Omega_{0} \subset \mathbb{R}^{d}$, $d=2,3$. The motion of the myocyte is defined using the smooth one-to-one deformation map $\psi$ : $\left.\Omega_{0} \times\right] 0, T\left[\rightarrow \Omega_{t} \times\right] 0, T[, \psi=\psi(\xi, t)$ representing the forward characteristics of the spatial velocity field $\boldsymbol{u}(\boldsymbol{x}, t) \equiv \partial_{t} \boldsymbol{\psi}$. The inverse deformation map is denoted by $\left.\boldsymbol{\xi}: \Omega_{t} \times\right] 0, T\left[\rightarrow \Omega_{0} \times\right] 0, T[$, $\boldsymbol{\xi}=\boldsymbol{\xi}(\boldsymbol{x}, t)$ and it represents the backward characteristics field satisfying

$$
\partial_{t} \xi+\boldsymbol{u} \cdot \nabla \boldsymbol{\xi}=\mathbf{0}, \quad \xi(t=0)=\boldsymbol{x},
$$

whereas the transport of the displacement field $\boldsymbol{d}=\boldsymbol{x}-\boldsymbol{\xi}$ reads

$$
\partial_{t} \boldsymbol{d}+\boldsymbol{u} \cdot \nabla \boldsymbol{d}=\boldsymbol{u}, \quad \boldsymbol{d}(t=0)=\mathbf{0} .
$$

From the deformation gradient tensor $\mathbf{F}=\operatorname{Grad} \psi$, we derive the right and left Cauchy-Green deformation tensors $\mathbf{C}=\mathbf{F}^{T} \mathbf{F}, \mathbf{B}=\mathbf{F F}^{T}$, and $J=\operatorname{det} \mathbf{F}>0$. Here, Grad $=\left(\partial / \partial \xi_{k} \mathbf{e}_{k}\right)_{k}$ stands for the material gradient, whereas $\nabla=\left(\partial / \partial x_{k} \mathbf{e}_{k}\right)_{k}$ and $\operatorname{div}(\cdot)$ denote, respectively, the gradient and divergence operators with respect to spatial coordinates. The symmetric part of a tensor $\mathbf{A}$ will be denoted by $\operatorname{sym}(\mathbf{A})=\mathbf{A}+\mathbf{A}^{T}$. We denote the inverse deformation gradient by $\boldsymbol{g}=\nabla \boldsymbol{\xi}$. One can readily see that $\operatorname{Grad} \boldsymbol{\psi}(\boldsymbol{\xi}, t) \nabla \boldsymbol{\xi}(\boldsymbol{\psi}, t)=\mathbf{I}$ and as consequence, the left Cauchy-Green tensor can be rewritten in terms of Eulerian quantities as $\mathbf{B}=\boldsymbol{g}^{-1} \boldsymbol{g}^{-T}=\nabla \xi^{-1} \nabla \xi^{-T}$.

An Eulerian description of motion requires to recover the deformation history everywhere in $\Omega_{t}$, which is typically represented by the material time derivative of the deformation gradient. However, there is no guarantee that the transported deformation gradient $\mathbf{F}$ will still describe a physical motion. On the other hand, the transport of the inverse characteristics $\xi$ by (2.1) or the Eulerian displacements $\boldsymbol{d}$ by (2.2) gives rise to difficulties when $\operatorname{det}(\nabla \boldsymbol{\xi})$ vanishes locally (especially in the case of large deformations such as rotation of a free solid). Even if some remedies are available (for instance, introducing penalization terms involving the curl of the deformation gradient [29]), here, we advect $\mathbf{B}$ (which is more natural in a fully Eulerian setting) using

$$
\partial_{t} \mathbf{B}+\boldsymbol{u} \cdot \nabla \mathbf{B}=\operatorname{sym}(\nabla \boldsymbol{u} \mathbf{B}),
$$

which follows from the relations:

$$
\partial_{t}\left(\nabla \xi^{-1}\right)+\boldsymbol{u} \cdot \nabla\left(\nabla \xi^{-1}\right)=\nabla \boldsymbol{u}\left(\nabla \xi^{-1}\right), \quad \partial_{t}\left(\nabla \boldsymbol{\xi}^{-T}\right)+\boldsymbol{u} \cdot \nabla\left(\nabla \boldsymbol{\xi}^{-T}\right)=\left(\nabla \boldsymbol{\xi}^{-T}\right) \nabla \boldsymbol{u}^{T} .
$$

\subsection{Anisotropic cardiac elastic response}

The properties of hyperelastic materials are characterized by scalar stored-energy functions. We will employ an anisotropic law whose strain energy is

$$
\mathcal{W}(\mathbf{F})=\frac{a}{2 b} \exp \left(b\left[I_{1}-d\right]\right)+\sum_{i=f, s} \frac{a_{i}}{2 b_{i}}\left[\exp \left(b_{i}\left[I_{4, i}-1\right]^{2}\right)-1\right]+\frac{a_{f s}}{2 b_{f_{s}}} \exp \left(b_{f_{s}} I_{8, f s}^{2}-1\right),
$$

where $a, a_{f}, a_{s}, a_{f s}, b, b_{f}, b_{s}$, and $b_{f_{s}}$ are experimentally fitted material parameters (taken as in [30], Table I) and the invariants $I_{i}$ are defined as follows

$$
I_{1}:=\operatorname{tr}(\mathbf{B}), I_{4, f}:=\boldsymbol{f} \cdot \boldsymbol{f}, I_{4, s}:=\boldsymbol{s} \cdot \boldsymbol{s}, I_{8, f s}:=\boldsymbol{f} \cdot \boldsymbol{s} .
$$


Table I. Values for the material, calcium dynamics, and activation parameters.

\begin{tabular}{lccccccccc}
\hline$a$ & $a_{f}$ & $a_{s}$ & $a_{f s}$ & $b$ & $b_{f}$ & $b_{s}$ & $b_{f s}$ & $d_{1}$ & $d_{2}$ \\
\hline $0.496 \mathrm{KPa}$ & $15.193 \mathrm{KPa}$ & $3.283 \mathrm{KPa}$ & 7.209 & 20.417 & 11.176 & $0.662 \mathrm{KPa}$ & 9.466 & $0.5 \frac{1}{\mu \mathrm{Ms}}$ & $2.5 \frac{1}{s}$ \\
$\nu_{1}$ & $\nu_{2}$ & $\nu_{3}$ & $v_{4}$ & $v_{5}$ & $k_{2}$ & $k_{3}$ & $k_{4}$ & $D_{f}$ & $D_{s}$ \\
\hline $1.58 \frac{\mu \mathrm{M}}{\mathrm{s}}$ & $16 \frac{\mu \mathrm{M}}{\mathrm{s}}$ & $91 \frac{\mu \mathrm{M}}{\mathrm{s}}$ & $2 s^{-1}$ & $0.2 s^{-1}$ & $1 \mu \mathrm{M}$ & $4 \mu \mathrm{M}^{2}$ & $0.7481 \mu \mathrm{M}^{4}$ & $60 \frac{\mu \mathrm{m}^{2}}{\mathrm{~s}}$ & $30 \frac{\mu \mathrm{m}^{2}}{\mathrm{~s}}$ \\
\hline
\end{tabular}
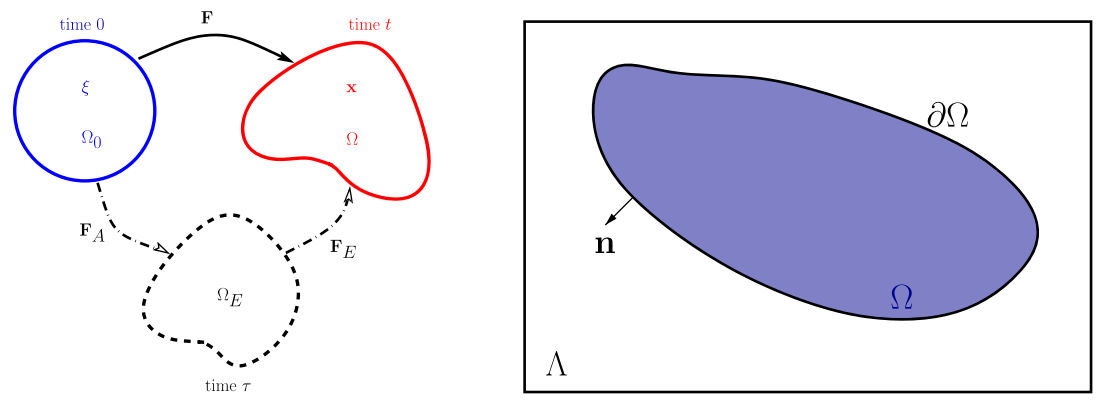

Figure 1. Left, multiplicative decomposition of the deformation gradient. Right, schematic representation of an elastic body $\Omega=\Omega_{t}$ immersed in an incompressible fluid domain $\Lambda \backslash \Omega_{t}$.

Let $\boldsymbol{f}_{0}$ and $\boldsymbol{s}_{0}$ denote the principal and secondary orthonormal axes, and let $\boldsymbol{f}:=\mathbf{F} \boldsymbol{f}_{0}, \boldsymbol{s}:=\mathbf{F} \boldsymbol{s}_{0}$ denote vectors (not necessarily orthonormal) representing the alignment of the axes in the deformed configuration. The Cauchy stress tensor is then specified as ( Appendix A):

$$
\begin{aligned}
\mathbf{T}= & \left(\frac{\partial \mathcal{W}}{\partial I_{1}} \frac{\partial I_{1}}{\partial \mathbf{F}}+\frac{\partial \mathcal{W}}{\partial I_{4, f}} \frac{\partial I_{4, f}}{\partial \mathbf{F}}+\frac{\partial \mathcal{W}}{\partial I_{4, s}} \frac{\partial I_{4, s}}{\partial \mathbf{F}}+\frac{\partial \mathcal{W}}{\partial I_{8, f s}} \frac{\partial I_{8, f_{s}}}{\partial \mathbf{F}}\right) \mathbf{F}^{T} \\
= & a \exp \left(b\left[I_{1}-d\right]\right) \mathbf{B}+2 a_{f}\left(I_{4, f}-1\right) \exp \left(b_{f}\left[I_{4, f}-1\right]^{2}\right) \boldsymbol{f} \otimes \boldsymbol{f} \\
& +2 a_{s}\left(I_{4, s}-1\right) \exp \left(b_{s}\left[I_{4, s}-1\right]^{2}\right) \boldsymbol{s} \otimes \boldsymbol{s}+2 a_{f_{s}} I_{8, f_{s}} \exp \left(b_{f_{s}} I_{8, f s}^{2}\right) \operatorname{sym}(\boldsymbol{f} \otimes \boldsymbol{s}) .
\end{aligned}
$$

The mechanical activation of the tissue is modeled using an active strain theory [26], where the deformation gradient is split into a passive and an active components $\mathbf{F}=\mathbf{F}_{\mathrm{E}} \mathbf{F}_{\mathrm{A}}$ (Figure 1, left). This represents that an intermediate elastic configuration $\Omega_{E}$ exists between the reference and current ones, where the strain energy function is rewritten in terms of $\mathbf{F}_{\mathrm{E}}=\mathbf{F F}_{\mathrm{A}}^{-1}$, then pulled back to the reference configuration, and finally written in terms of the following modified invariants

$$
\begin{aligned}
& I_{1}^{\mathrm{E}}:=\operatorname{tr}\left(\mathbf{B}_{\mathrm{E}}\right)=I_{1}-\sum_{l \in\{f, s, n\}} \frac{\gamma_{1}\left(\gamma_{1}+2\right)}{\left(\gamma_{1}+1\right)^{2}} \mathbf{F} \boldsymbol{l}_{0} \cdot \mathbf{F} \boldsymbol{l}_{0}, I_{8, f s}^{\mathrm{E}}:=\frac{1}{1+\gamma_{\mathrm{f}}} \boldsymbol{f} \cdot \frac{1}{1+\gamma_{\mathrm{s}}} \boldsymbol{s}=\frac{I_{8, f_{s}}}{\left(1+\gamma_{\mathrm{f}}\right)\left(1+\gamma_{\mathrm{s}}\right)}, \\
& I_{4, l}^{\mathrm{E}}:=\mathbf{F}_{\mathrm{E}} \boldsymbol{l}_{0} \cdot \mathbf{F}_{\mathrm{E}} \boldsymbol{l}_{0}=\mathbf{F F}_{\mathrm{A}}^{-1} \boldsymbol{l}_{0} \cdot \mathbf{F F}_{\mathrm{A}}^{-1} \boldsymbol{l}_{0}=\left(1-\frac{\gamma_{1}}{1+\gamma_{l}}\right)^{2} \boldsymbol{l} \cdot \boldsymbol{l}=\left(1+\gamma_{1}\right)^{-2} I_{4, l}, \quad l=f, s, n .
\end{aligned}
$$

Here, $\gamma_{1}$ is a smooth scalar activation function (to be defined later) acting on the generic direction $\boldsymbol{l}_{0}$, whereas the active deformation gradient has a general anisotropic distribution on a triplet of orthonormal vectors $\mathbf{F}_{\mathrm{A}}=\mathbf{I}+\gamma_{\mathrm{f}} \boldsymbol{f}_{0} \otimes \boldsymbol{f}_{0}+\gamma_{\mathrm{s}} \boldsymbol{s}_{0} \otimes \boldsymbol{s}_{0}+\gamma_{\mathrm{n}} \boldsymbol{n}_{0} \otimes \boldsymbol{n}_{0}$. 
After writing explicitly the derivatives of $\mathcal{W}$ with respect to the modified invariants (Appendix A), the activated stress tensor reads

$$
\begin{aligned}
\mathbf{T}= & a \exp \left(b\left[I_{1}^{\mathrm{E}}-d\right]\right)\left(\mathbf{B}-\sum_{l \in\{f, s\}} \frac{\gamma_{1}\left(\gamma_{1}+2\right)}{\left(\gamma_{1}+1\right)^{2}} \boldsymbol{l} \otimes \boldsymbol{l}\right)+\frac{2 a_{f}}{\left(1+\gamma_{\mathrm{f}}\right)^{2}}\left(I_{4, f}^{\mathrm{E}}-1\right) \exp \left(b_{f}\left[I_{4, f}^{\mathrm{E}}-1\right]^{2}\right) \boldsymbol{f} \otimes \boldsymbol{f} \\
& +\frac{2 a_{s}}{\left(1+\gamma_{\mathrm{s}}\right)^{2}}\left(I_{4, s}^{\mathrm{E}}-1\right) \exp \left(b_{s}\left[I_{4, s}^{\mathrm{E}}-1\right]^{2}\right) \boldsymbol{s} \otimes \boldsymbol{s} \\
& +\frac{2 a_{f s}}{\left(1+\gamma_{\mathrm{f}}\right)\left(1+\gamma_{\mathrm{s}}\right)} I_{8, f s}^{\mathrm{E}} \exp \left(b_{f s}\left[I_{8, f s}^{\mathrm{E}}\right]^{2}\right) \operatorname{sym}(\boldsymbol{f} \otimes s) .
\end{aligned}
$$

\subsection{Calcium dynamics and calcium-driven mechanical activation}

Intracellular calcium kinetics can be described with a number of models of varied complexity (see for instance [31-34]). Since our interest is to recover cytosolic transients of calcium, it is enough to consider reaction-diffusion models based on the interaction of cytosolic and sarcoplasmic calcium concentrations, $w_{c}$ and $w_{s}$, respectively. The following specifications are used ([32]):

$$
\left.\partial_{t} w_{c}+\boldsymbol{u} \cdot \nabla w_{c}-\operatorname{div}\left(\mathbf{D}(\boldsymbol{x}) \nabla w_{c}\right)=K\left(w_{c}, w_{s}\right), \quad \partial_{t} w_{s}+\boldsymbol{u} \cdot \nabla w_{s}=L\left(w_{c}, w_{s}\right) \quad \text { in } \Omega_{t} \times\right] 0, T[,
$$

where $\mathbf{D}=\operatorname{diag}\left(D_{f}, D_{s}\right)$ and the nonlinear reaction terms are given by

$$
\begin{aligned}
& K\left(w_{c}, w_{s}\right)=v_{1}-\frac{v_{2} w_{c}^{2}}{k_{2}+w_{c}^{2}}+\frac{v_{3} w_{c}^{4} w_{s}^{2}}{\left(k_{3}+w_{s}^{2}\right)\left(k_{4}+w_{c}^{4}\right)}-v_{4} w_{c}, \\
& L\left(w_{c}, w_{s}\right)=\frac{v_{2} w_{c}^{2}}{k_{2}+w_{c}^{2}}-\frac{v_{3} w_{c}^{4} w_{s}^{2}}{\left(k_{3}+w_{s}^{2}\right)\left(k_{4}+w_{c}^{4}\right)}-v_{5} w_{s} .
\end{aligned}
$$

The process of mechanical activation involves complex intracellular dynamics that triggers the binding of calcium to troponin, and eventually resulting in the activation of thin filaments [5]. We can assume that such an activation depends directly on the cytosolic calcium concentration through the following equation (a spatial analog to the macroscopic calcium-dependent activation model proposed in [28]) $\partial_{t} \gamma_{\mathrm{f}}+\boldsymbol{w} \cdot \nabla \gamma_{\mathrm{f}}=G\left(w_{c}, \gamma_{\mathrm{f}}\right)$, with $G\left(w_{c}, \gamma_{\mathrm{f}}\right)=-d_{1} w_{c}-d_{2} \gamma_{\mathrm{f}}$, where the parameters have been tuned to match [32] and are set as in Table I. The behavior of these reaction kinetics is depicted in Figure 14, left.

\subsection{Level set formulation}

Let $T>0$ and for any $t \in] 0, T$ [ let us assume that the cell has smooth boundary $\Gamma=\partial \Omega_{t}$ and it is surrounded by a regular domain $\Lambda$ (Figure 1, right). The closed surface representing the fluidstructure interface is redefined as $\Gamma=\{(t, \boldsymbol{x}) \in] 0, T[\times \Lambda: \psi(t, \boldsymbol{x})=0\}$, where $\psi$ is a level set function. By convention, $\psi(t, \cdot)$ is negative in $\Omega_{t}$. The interface $\Gamma$ is transported with the same velocity, and thus the level set function satisfies

$$
\left.\partial_{t} \psi+\boldsymbol{w} \cdot \nabla \psi=0 \text { in }\right] 0, T[\times \Lambda,
$$

equipped with suitable boundary and initial data $\psi=\psi_{b}$ on $] 0, T\left[\times \Sigma_{-}\right.$and $\psi(0)=\psi_{0}$ in $\Lambda$, where $\Sigma_{-}=\{\boldsymbol{x} \in \partial \Lambda: \boldsymbol{u} \cdot \boldsymbol{v}(\boldsymbol{x})<0\}$ is the upstream (inflow) boundary and $\boldsymbol{v}$ denotes the outward unit normal to the surrounding domain $\Lambda$. The function $\psi_{0}$, acting as initial datum for the scalar transport equation, denotes the signed distance between $\boldsymbol{x}$ and the given initial cell membrane $\partial \Omega_{0}$ :

$$
\psi_{0}(x)=\left\{\begin{array}{cl}
\inf \left\{|y-x| ; y \in \partial \Omega_{0}\right\} & \text { if } x \notin \Omega_{0}, \\
-\inf \left\{|y-x| ; y \in \partial \Omega_{0}\right\} & \text { otherwise. }
\end{array}\right.
$$


The description of preferred directions can be naturally managed using a level set approach as in [23]. Let $\varphi_{0}^{f}, \varphi_{0}^{s}: \Omega_{0} \rightarrow \mathbb{R}$ be signed distance functions such that the preferred directions are given respectively by $\boldsymbol{f}_{0}(\boldsymbol{\xi})=\nabla \varphi_{0}^{f}(\xi)$ and $\boldsymbol{s}_{0}(\boldsymbol{\xi})=\nabla \varphi_{0}^{s}(\boldsymbol{\xi})$, and let us denote by $\varphi^{f}(\boldsymbol{x}, t)=\varphi_{0}^{f}(\boldsymbol{\xi}(\boldsymbol{x}, t), t), \varphi^{s}(\boldsymbol{x}, t)=\varphi_{0}^{s}(\boldsymbol{\xi}(\boldsymbol{x}, t), t)$ the corresponding transported fields. Notice that $\nabla \varphi^{f}=\nabla \xi^{T} \nabla \varphi_{0}^{f}$ and $\nabla \varphi^{s}=\nabla \xi^{T} \nabla \varphi_{0}^{s}$, which yields that the principal direction in the current configuration assumes the form

$$
\boldsymbol{f}=\mathbf{F} \boldsymbol{f}_{0}=\mathbf{F F}^{T} \mathbf{F}^{-T} \nabla \varphi_{0}^{f}=\mathbf{B} \boldsymbol{g}^{T} \nabla \varphi_{0}^{f}=\mathbf{B} \nabla \varphi^{f},
$$

and analogously for the secondary direction $\boldsymbol{s}=\mathbf{F} \nabla \varphi_{0}^{s}=\mathbf{B} \nabla \varphi^{s}$.

\subsection{Redistancing and regularization}

Problem (2.7) does not preserve the so-called distance function property of the initial level set, which may lead to numerical instabilities when the level set gradient is either very high or very small, often occurring near $\Gamma$ and $\partial \Lambda$ (see, e.g., [35,36]). The solution of an auxiliary problem (called redistancing method, [21,37]) permits to re-establish the signed distance property of the level set function while maintaining the position of the zero-level-set; however, it may introduce a substantial loss of mass. Therefore, we introduce a Lagrange multiplier in the vicinity of the interface to enforce local mass conservation, which also improves the performance of the method ([38]).

Let $\tilde{\psi}(t, \cdot)$ be the solution of $(2.7)$. Introducing a pseudo-time $\tau$, we look for the solution of

$$
\left\{\begin{aligned}
\frac{\partial \psi}{\partial \tau}(\tau, \boldsymbol{x} ; t)+\boldsymbol{v} \cdot \nabla \psi(\tau, \boldsymbol{x} ; t) & =\operatorname{sgn}(\tilde{\psi}(t, \boldsymbol{x}))+\lambda(\tau, x ; t) g(\psi(\tau, \boldsymbol{x} ; t)) & & \text { a.e. in }] 0,+\infty[\times \Lambda, \\
\psi(0, \boldsymbol{x} ; t) & =\tilde{\psi}(t, \boldsymbol{x}) & & \text { a.e. in } \Lambda .
\end{aligned}\right.
$$

The advection vector is $v=\operatorname{sgn}(\tilde{\psi}) \frac{\nabla \psi}{|\nabla \psi|}$, and $\operatorname{sgn}(\tilde{\varphi})$ assumes the values $0,-1,+1$ on $\partial \Omega_{t}, \Omega_{t}$, and $\Lambda \backslash \Omega_{t}$, respectively. The Lagrange multiplier $\lambda(\tau, \boldsymbol{x} ; t)$ locally enforces a constant volume at $\boldsymbol{x} \in \Lambda$, whereas $g(\psi)$ localizes the mass correction near the interface (and consequently depends on the Dirac measure $\delta(\psi)$ ). Moreover, the Lagrange multiplier has an explicit average value $\lambda_{\mathcal{V}}$ over an arbitrary finite volume or patch $\mathcal{V} \subset \Lambda$ :

$$
\lambda_{\mathcal{V}}(\tau ; t)=\frac{\int_{\mathcal{V}} \delta(\psi)(\boldsymbol{v} \cdot \nabla \psi-\operatorname{sgn}(\tilde{\varphi}))-x}{\int_{\mathcal{V}} \delta(\psi) g(\psi)}, \text { when } \mathcal{V} \cap \partial \Omega_{t} \neq \emptyset \text { and } 0 \text { otherwise. }
$$

This implies that the stationary solution satisfies $|\nabla \psi|=1$ a.e. in $\Lambda$, and therefore $\psi(\infty, \cdot ; t)$ is a signed distance and can be taken as the new level set function $\varphi(t, \cdot)$. An advantage of this procedure is that the solution of (2.9) preserves the position of $\partial \Omega_{t}$ and therefore the volume $\left|\Omega_{t}\right|$ for any $\tau>0$.

For a given $\varepsilon>0$, we introduce a layer of width $2 \varepsilon$ around $\Gamma$, where the regularization parameter is chosen proportional to the local mesh-size. The sign, Heaviside and Dirac functions are then replaced by their regularized versions, defined for all $\psi \in \mathbb{R}$ as

$$
\begin{gathered}
\operatorname{sgn}_{\varepsilon}(\psi)=2 \mathscr{H}_{\varepsilon}(\psi)-1, \quad \mathscr{H}_{\varepsilon}(\psi)= \begin{cases}0, & \text { if } \psi<-\varepsilon, \\
\frac{1}{2}\left(1+\frac{\psi}{\varepsilon}+\pi^{-1} \sin \left(\frac{\pi \psi}{\varepsilon}\right)\right), & \text { if }|\psi| \leqslant \varepsilon, \\
1, & \text { otherwise }\end{cases} \\
\delta_{\varepsilon}(\psi)=\frac{\mathrm{d} \mathscr{H}_{\varepsilon}}{\mathrm{d} \psi}(\psi)=\frac{1}{2 \varepsilon}\left(1+\cos \left(\frac{\pi \psi}{\varepsilon}\right)\right), \quad \text { if }|\psi| \leqslant \varepsilon \text { and } 0, \text { otherwise. }
\end{gathered}
$$

The density assumes constant values $\rho^{\text {sd }}$ and $\rho^{\text {fd }}$ inside and outside $\Omega_{t}$, i.e., $\rho(\psi)=\rho^{\text {sd }} \mathscr{H}(\psi)+$ $\rho^{\text {fd }}(1-\mathscr{H}(\psi))$, and its regularized form is $\rho_{\varepsilon}(\psi)=\rho^{\text {sd }} \mathscr{H}_{\varepsilon}(\psi)+\rho^{\mathbf{f d}}\left(1-\mathscr{H}_{\varepsilon}(\psi)\right)$. 


\subsection{Final form of the nonlinear coupled problem}

If we suppose fluid incompressibility, the stresses in the solid and fluid domains are $\sigma^{\text {sd }}=$ $\mathbf{T}\left(\mathbf{B}, \gamma_{\mathrm{f}}\right)-p \mathbf{I}$ and $\boldsymbol{\sigma}^{\mathbf{f d}}=2 \mu \boldsymbol{\varepsilon}(\boldsymbol{w})-p \mathbf{I}$, respectively, where $p=p(\boldsymbol{x})$ is pressure, $\mu$ is the dynamic viscosity of the external fluid, and $\boldsymbol{\varepsilon}(\boldsymbol{w})=\left(\nabla \boldsymbol{w}+\nabla \boldsymbol{w}^{T}\right) / 2$ is the strain rate tensor. The regularized total stress is $\boldsymbol{\sigma}\left(\boldsymbol{w}, \mathbf{B}, \gamma_{\mathrm{f}}, p\right)=\mathscr{H}_{\varepsilon}(\psi) \mathbf{T}\left(\mathbf{B}, \gamma_{\mathrm{f}}\right)+\left(1-\mathscr{H}_{\varepsilon}(\psi)\right) 2 \mu \boldsymbol{\varepsilon}(\boldsymbol{w})-p \mathbf{I}$. Instead of considering a particular pressure field defined on each medium and artificially extend them to the whole domain, we here assume a continuous pressure everywhere (as in other contributions in Eulerian elasticity $[15,22,23])$. This simplification does not have a major influence on the accuracy of the method and therefore other alternatives may be used [13]. Collecting the elements aforementioned, we arrive at the following fluid-structure interaction coupled model describing the bulk equilibrium and balance of different fluxes: Find $\psi, \mathbf{B}, \boldsymbol{w}, p, \varphi^{f}, \varphi^{s}, w_{c}, w_{s}$ and $\gamma_{\mathrm{f}}$ such that, in $] 0, T[\times \Lambda$,

$$
\begin{aligned}
\partial_{t} \psi+\boldsymbol{u} \cdot \nabla \psi & =0 \\
\partial_{t} \mathbf{B}+\boldsymbol{u} \cdot \nabla \mathbf{B} & =\operatorname{sym}(\nabla \boldsymbol{u} \mathbf{B}) \\
\rho_{\varepsilon}(\psi)\left(\partial_{t} \boldsymbol{u}+\boldsymbol{u} \cdot \nabla \boldsymbol{w}\right)-\operatorname{div} \boldsymbol{\sigma} & =0 \\
\operatorname{div} \boldsymbol{u} & =0 \\
\partial_{t} \varphi^{f}+\boldsymbol{u} \cdot \nabla \varphi^{f} & =0 \\
\partial_{t} \varphi^{s}+\boldsymbol{u} \cdot \nabla \varphi^{s} & =0 \\
\partial_{t} w_{c}+\boldsymbol{w} \cdot \nabla w_{c}-\operatorname{div}\left(\mathbf{D}(\boldsymbol{x}) \nabla w_{c}\right) & =K\left(w_{c}, w_{s}\right) \\
\partial_{t} w_{s}+\boldsymbol{u} \cdot \nabla w_{s} & =L\left(w_{c}, w_{s}\right) \\
\partial_{t} \gamma_{\mathrm{f}}+\boldsymbol{u} \cdot \nabla \gamma_{\mathrm{f}} & =G\left(\boldsymbol{u}, \gamma_{\mathrm{f}}\right) .
\end{aligned}
$$

Boundary conditions are set as

$$
\begin{aligned}
& \left.\psi=\psi_{b} \text { on }\right] 0, T\left[\times \Sigma_{-}, \boldsymbol{v} \cdot\left(\mathbf{D}(\boldsymbol{x}) \nabla w_{c}\right)=\mathbf{0} \text { on }\right] 0, T[\times \partial \Lambda, \\
& \boldsymbol{u}=\mathbf{0} \text { on }] 0, T\left[\times \Sigma_{D},(2 \eta \boldsymbol{\varepsilon}(\boldsymbol{u})-p \mathbf{I}) \boldsymbol{v}=\mathbf{0} \text { on }\right] 0, T\left[\times \Sigma_{N},\right.
\end{aligned}
$$

where $\Sigma_{D}$ and $\Sigma_{N}$ represent portions of the boundary where either Dirichlet or stress-free boundary conditions are enforced, respectively. Initial data are set as

$\left(\boldsymbol{u}(0), \psi(0), \mathbf{B}(0), \varphi^{f}(0), \varphi^{s}(0), w_{c}(0), w_{s}(0), \gamma_{\mathrm{f}}(0)\right)=\left(\boldsymbol{u}_{0}, \psi_{0}, \mathbf{I}, \varphi_{0}^{f}, \varphi_{0}^{s}, w_{c, 0}, w_{s, 0}, \gamma_{f, 0}\right), \quad$ in $\Lambda$.

Equations (2.10e)-(2.10f) associated to the preferred directions may also be computed using the backward characteristics by $\varphi^{f}(\boldsymbol{x}, t)=\varphi_{0}^{f}(\boldsymbol{\xi}(\boldsymbol{x}, t))$ and $\varphi^{s}(\boldsymbol{x}, t)=\varphi_{0}^{s}(\boldsymbol{\xi}(\boldsymbol{x}, t))$, respectively.

\section{NUMERICAL APPROXIMATION}

\subsection{Time discretization}

Let us divide $[0, T]$ into $N$ subintervals $\left(t_{n}, t_{n+1}\right), n=0, \ldots, N-1$ of constant step $\Delta t$. For any $n \geqslant 1$, the unknowns $\psi_{n}, \mathbf{B}_{n}, \boldsymbol{w}_{n}, p_{n}, \varphi_{n}^{f}, \varphi_{n}^{s}, w_{c, n}, w_{s, n}$ and $\gamma_{n}$ at time step $n$ are computed iteratively. We employ the method of characteristics only for the advection of the level set functions and calcium-driven activation. For $0<s<T$, the characteristic curve $\chi(\cdot, x, t)$ passing at time $t$ through $\boldsymbol{x} \in \Lambda$ is defined as the solution of

$$
\partial_{t} \chi(s, \boldsymbol{x} ; t)=\boldsymbol{w}(\chi(s, \boldsymbol{x} ; t), t), \quad \chi(t, \boldsymbol{x} ; t)=\boldsymbol{x} .
$$


For any function $\varphi(t, \boldsymbol{x})$, the total derivative reads

$$
\left(\partial_{t} \varphi+\boldsymbol{u} \cdot \nabla \varphi\right)(t, x)=\left.\frac{\partial}{\partial \tau}(\varphi(\chi(t, x ; \tau), \tau))\right|_{\tau=t} .
$$

Following [39], this derivative is approximated by a first-order backward Euler scheme:

$$
\frac{D \varphi}{D t}\left(t_{n}, x\right) \approx \frac{\varphi\left(t_{n}, x\right)-\varphi\left(t_{n-1}, \chi_{n-1}^{(1)}(x)\right)}{\Delta t},
$$

where $\chi_{n-1}^{(1)}(x)=x-\Delta t \boldsymbol{u}_{n-1}(x)$ denotes the first-order approximation of $\chi\left(t_{n-1}, x ; t_{n}\right)$ and the solid shape at step $n$ is $\Omega_{n}:=\left\{x \in \Lambda ; \varphi_{n}(x)<0\right\}$. Problem (2.10) then reads: Find $\psi_{n}, \mathbf{B}_{n}, \boldsymbol{u}_{n}, p_{n}$, $\varphi_{n}^{f}, \varphi_{n}^{s}, w_{c, n}, w_{s, n}$ and $\gamma_{n}$ such that, everywhere,

$$
\begin{aligned}
\psi_{n}-\psi_{n-1} \circ \chi_{n-1}^{(1)} & =0 \\
\left(\frac{\mathbf{B}_{n}-\mathbf{B}_{n-1}}{\Delta t}\right)+\boldsymbol{u}_{n} \cdot \nabla \mathbf{B}_{n} & =\operatorname{sym}\left(\nabla \boldsymbol{u}_{n} \mathbf{B}_{n}\right) \\
\rho\left(\psi_{n}\right)\left(\frac{\boldsymbol{u}_{n}-\boldsymbol{u}_{n-1}}{\Delta t}+\boldsymbol{u}_{n} \cdot \nabla \boldsymbol{u}_{n}\right)-\operatorname{div} \boldsymbol{\sigma}_{n} & =0 \\
\operatorname{div} \boldsymbol{u}_{n} & =0 \\
\varphi_{n}^{f}+\Delta t \boldsymbol{u}_{n} \cdot \nabla \varphi_{n}^{f} & =\varphi^{f, n-1} \\
\varphi_{n}^{s}+\Delta t \boldsymbol{u}_{n} \cdot \nabla \varphi_{n}^{s} & =\varphi^{s, n-1} \\
w_{c, n}-w_{c, n-1} \circ \chi_{n-1}^{(1)}-\Delta t \operatorname{div}\left(\mathbf{D}(\boldsymbol{x}) \nabla w_{c, n}\right) & =\Delta t K\left(w_{c, n-1}, w_{s, n-1}\right) \\
w_{s, n}-w_{s, n-1} \circ \chi_{n-1}^{(1)} & =\Delta t L\left(w_{c, n-1}, w_{s, n-1}\right) \\
\gamma_{n}-\gamma_{f, n-1} \circ \chi_{n-1}^{(1)} & =\Delta t G\left(w_{c, n-1}, \gamma_{f, n-1}\right) .
\end{aligned}
$$

\subsection{Consistent linearization and Newton algorithm}

We will drop the subscript $n$ whenever it is clear from the context. We introduce the functional spaces $V:=\left\{\boldsymbol{v} \in H^{1}(\Lambda)^{d}: \boldsymbol{v}=\mathbf{0}\right.$ on $\left.\Sigma_{D}\right\}, Q=L^{2}(\Lambda), M:=\left\{\boldsymbol{\tau} \in\left(L^{2}(\Lambda)\right)^{d \times d}: \boldsymbol{\tau}=\boldsymbol{\tau}^{T}\right\}$ and the weighted multi-linear forms

$$
\begin{aligned}
m(\boldsymbol{u}, \boldsymbol{v} ; w) & =\int_{\Lambda} w \boldsymbol{u} \cdot \boldsymbol{v}, a(\boldsymbol{u}, \boldsymbol{v} ; \boldsymbol{w})=\int_{\Lambda} 2 w \boldsymbol{\varepsilon}(\boldsymbol{u}): \boldsymbol{\varepsilon}(\boldsymbol{v}), b(\boldsymbol{v}, q)=-\int_{\Lambda} q \operatorname{div} \boldsymbol{u}, \\
c(\boldsymbol{u}, \boldsymbol{v} ; w, \boldsymbol{w}) & =\int_{\Lambda} w[(\boldsymbol{u} \cdot \nabla) \boldsymbol{w}+(\boldsymbol{w} \cdot \nabla) \boldsymbol{u}] \cdot \boldsymbol{v}, \quad d(\boldsymbol{u}, \boldsymbol{v} ; w, \mathbf{B})=\int_{\Lambda} w(\mathbf{B}: \nabla \boldsymbol{u})(\mathbf{B}: \nabla \boldsymbol{v}), \\
e(\boldsymbol{u}, \boldsymbol{v} ; w, \mathbf{B}) & =\int_{\Lambda} w \operatorname{sym}(\nabla \boldsymbol{u} \mathbf{B}): \nabla \boldsymbol{v}, \quad f(\boldsymbol{u}, \mathbf{B} ; w)=\int_{\Lambda} w \mathbf{B}: \boldsymbol{\varepsilon}(\boldsymbol{u}), \quad g(\boldsymbol{\tau}, \boldsymbol{\gamma})=\int_{\Lambda} \boldsymbol{\tau}: \boldsymbol{\gamma}, \\
\alpha(\mathbf{B}, \boldsymbol{\tau} ; \boldsymbol{u}) & =\int_{\Lambda}[(\boldsymbol{u} \cdot \nabla) \mathbf{B}-\operatorname{sym}(\nabla \boldsymbol{u B})]: \boldsymbol{\tau}, \beta(\boldsymbol{u}, \boldsymbol{\tau} ; \mathbf{B})=\alpha(\mathbf{B}, \boldsymbol{\tau} ; \boldsymbol{u}), i(\phi, \xi)=\int_{\Lambda} \phi \xi, \\
l(\phi, \xi ; \mathbf{D}) & =\int_{\Lambda} \mathbf{D} \nabla \phi \cdot \nabla \xi,
\end{aligned}
$$


defined for all $\boldsymbol{w}, \boldsymbol{v}, \boldsymbol{w} \in V ; q \in Q ; \boldsymbol{\tau}, \boldsymbol{\gamma} \in M ; \phi, \xi \in H^{1}(\Lambda)$ and $\mathbf{D} \in \mathbb{R}^{d \times d}$, and for any $w \in L^{\infty}(\Lambda), \mathbf{B} \in\left(L^{\infty}(\Lambda)\right)^{d \times d}$. The linearization of the stress in the direction of a velocity and pressure increments $\delta \boldsymbol{w}, \delta p$, is detailed in Appendix A. The tangent problem associated to (3.2),(3.3),(3.4) reads: Find $\left(\delta \boldsymbol{w}^{k}, \delta p^{k}, \delta \mathbf{B}^{k}\right) \in V \times Q \times M$ such that

$$
\begin{aligned}
& m\left(\delta \boldsymbol{u}^{k}, \boldsymbol{v} ; \frac{\rho_{\varepsilon}\left(\psi_{n-1}\right)}{\Delta t}\right)+c\left(\delta \boldsymbol{u}^{k}, \boldsymbol{v} ; \rho_{\varepsilon}\left(\psi_{n-1}\right), \boldsymbol{u}^{k}\right)+a\left(\delta \boldsymbol{u}^{k}, \boldsymbol{v} ; 1-\mathscr{H}_{\varepsilon}\left(\psi_{n-1}\right)\right) \\
& +b\left(\boldsymbol{v}, \delta p^{k}\right)+d\left(\delta \boldsymbol{u}^{k}, \boldsymbol{v} ; 4 b \psi_{1}^{\mathrm{E}, k} \mathscr{H}_{\varepsilon}\left(\psi_{n-1}\right), \hat{\mathbf{B}}_{1}^{k}\right) \\
& +e\left(\delta \boldsymbol{u}^{k}, \boldsymbol{v} ; 2 \psi_{1}^{\mathrm{E}, k} \mathscr{H}_{\varepsilon}\left(\psi_{n-1}\right), \hat{\mathbf{B}}_{1}^{k}\right) \\
& +\sum_{l \in\{f, s\}}\left(d\left(\delta \boldsymbol{u}^{k}, \boldsymbol{v} ; \zeta_{4, l}^{\mathrm{E}, k} \mathscr{H}_{\varepsilon}\left(\psi_{n-1}\right), \hat{\mathbf{B}}_{4, l}^{k}\right)\right. \\
& \left.+e\left(\delta \boldsymbol{u}^{k}, \boldsymbol{v} ; \frac{2 \psi_{4, l}^{\mathrm{E}, k}}{\left(1+\gamma_{l, n-1}\right)^{2}} \mathscr{H}_{\varepsilon}\left(\psi_{n-1}\right), \hat{\mathbf{B}}_{4, l}^{k}\right)\right) \\
& +d\left(\delta \boldsymbol{u}^{k}, \boldsymbol{v} ; \zeta_{8, f s}^{\mathrm{E}, k} \mathscr{H}_{\varepsilon}\left(\psi_{n-1}\right), \hat{\mathbf{B}}_{8, f_{s}}^{k}\right) \\
& +e\left(\delta \boldsymbol{u}^{k}, \boldsymbol{v} ; \frac{\psi_{8, f s}^{\mathrm{E}, k}}{\left(1+\gamma_{f, n-1}\right)\left(1+\gamma_{s, n-1}\right)} \mathscr{H}_{\varepsilon}\left(\psi_{n-1}\right), \hat{\mathbf{B}}_{8, f s}^{k}\right) \\
& =-\left\langle\mathcal{R}_{\boldsymbol{u}, p}\left(\boldsymbol{u}^{k}, p^{k}\right), \boldsymbol{v}\right\rangle_{V, V^{\prime}}, b\left(\delta \boldsymbol{u}^{k}, q\right) \\
& b\left(\delta \boldsymbol{u}^{k}, q\right)=-\left\langle\mathcal{R}_{p}\left(\boldsymbol{u}^{k}\right), q\right\rangle_{Q, Q^{\prime}}, g\left(\frac{\delta \mathbf{B}^{k}}{\Delta t}, \boldsymbol{\tau}\right) \\
& g\left(\frac{\delta \mathbf{B}^{k}}{\Delta t}, \boldsymbol{\tau}\right)+\alpha\left(\delta \mathbf{B}^{k}, \boldsymbol{\tau} ; \boldsymbol{u}^{k}\right)+\beta\left(\delta \boldsymbol{u}^{k}, \boldsymbol{\tau} ; \mathbf{B}^{k}\right)=-\left\langle\mathcal{R}_{\boldsymbol{u}, \mathbf{B}}\left(\boldsymbol{u}^{k}, \mathbf{B}^{k}\right), \boldsymbol{\tau}\right\rangle_{M, M^{\prime}},
\end{aligned}
$$

for all $(\boldsymbol{v}, q, \boldsymbol{\tau}) \in V \times Q \times M$, where the corresponding residuals are defined as

$$
\begin{aligned}
\left\langle\mathcal{R}_{\boldsymbol{u}, p}\left(\boldsymbol{u}^{k}, p^{k}\right), \boldsymbol{v}\right\rangle= & m\left(\boldsymbol{u}^{k}-\boldsymbol{u}_{n-1}, \boldsymbol{v} ; \frac{\rho_{\varepsilon}\left(\psi_{n-1}\right)}{\Delta t}\right)+\frac{1}{2} c\left(\boldsymbol{u}^{k}, \boldsymbol{v} ; \rho_{\varepsilon}\left(\psi_{n-1}\right), \boldsymbol{u}^{k}\right) \\
& +a\left(\boldsymbol{u}^{k}, \boldsymbol{v} ; 1-\mathscr{H}_{\varepsilon}\left(\psi_{n-1}\right)\right)+b\left(\boldsymbol{v}, p^{k}\right) \\
& +f\left(\boldsymbol{u}^{k}, \mathscr{H}_{\varepsilon}\left(\psi_{n-1}\right) \mathbf{T}^{k}+\left(1-\mathscr{H}_{\varepsilon}\left(\psi_{n-1}\right)\right), 2 \mu \boldsymbol{\varepsilon}\left(\boldsymbol{u}^{k}\right) ; 1\right), \\
\left\langle\mathcal{R}_{p}\left(\boldsymbol{u}^{k}\right), q\right\rangle= & b\left(\boldsymbol{u}^{k}, q\right), \quad\left\langle\mathcal{R}_{\boldsymbol{u}, \mathbf{B}}\left(\boldsymbol{u}^{k}, \mathbf{B}^{k}\right), \boldsymbol{\tau}\right\rangle=g\left(\frac{\mathbf{B}^{k}-\mathbf{B}_{n}}{\Delta t}, \boldsymbol{\tau}\right)+\alpha\left(\mathbf{B}^{k}, \boldsymbol{\tau} ; \boldsymbol{u}^{k}\right),
\end{aligned}
$$

for all $v \in V, q \in Q, \tau \in M$. An alternative variational formulation, more convenient from the implementation viewpoint, is presented in Appendix B.

\subsection{Transport of principal directions, calcium, and activation}

The transport of $\left(\varphi_{l, n}\right)_{l \in\{f, s\}}$ is uncoupled from (3.10) but is still solved inside the Newton loop, because at $t_{n}$ the stress $\mathbf{T}_{n}^{k}$ depends on $\left(\varphi_{l, n}\right)_{l \in\{f, s\}}$. Then, for given $\left(\varphi_{l, n}^{0}=\varphi_{l, n-1}\right)_{l \in\{f, s\}}$ and 
$\boldsymbol{u}^{k+1}$, we look for $\left(\varphi_{l}^{k}\right)_{l \in\{f, s\}} \in Q$ such that

$$
i\left(\frac{\varphi_{l}^{k}}{\Delta t}, \xi\right)+j\left(\varphi_{l}^{k}, \xi ; \boldsymbol{u}^{k+1}\right)=i\left(\frac{\varphi_{l, n-1}}{\Delta t}, \xi\right) \text {, with } l \in\{f, s\}, \forall \xi \in Q
$$

The reaction-diffusion system governing the calcium-driven mechanical activation is discretized in time using a semi-implicit method. The variational formulation of (3.7)-(3.9) is as follows: given $w_{c, n-1}, w_{s, n-1}, \gamma_{f, n-1}$ and $w_{n}$, find $w_{c, n}, w_{s, n}$ and $\gamma_{f, n} \in H^{1}(\Lambda)$ such that

$$
\begin{aligned}
i\left(\frac{w_{c, n}}{\Delta t}, \xi\right)+l\left(w_{c, n}, \xi ; \mathbf{D}\right) & =i\left(\frac{w_{c, n-1} \circ \chi_{n-1}^{(1)}}{\Delta t}+K\left(w_{c, n-1}, w_{s, n-1}\right), \xi\right), \\
i\left(\frac{w_{s, n}}{\Delta t}, \xi\right) & =i\left(\frac{w_{s, n-1} \circ \chi_{n-1}^{(1)}}{\Delta t}+L\left(w_{c, n-1}, w_{s, n-1}\right), \xi\right), \\
i\left(\frac{\gamma_{f, n}}{\Delta t}, \xi\right) & =i\left(\frac{\gamma_{f, n-1} \circ \chi_{n-1}^{(1)}}{\Delta t}+G\left(w_{c, n-1}, \gamma_{f, n-1}\right), \xi\right) .
\end{aligned}
$$

The overall coupling method is detailed in Algorithm 1. Notice that the transport of the fluidstructure interface, activation, and calcium, are solved outside the Newton loop. The velocity field is extrapolated by a second order prediction given by

$$
\boldsymbol{u}_{n}^{*}=2 \boldsymbol{u}_{n-1}-\boldsymbol{u}_{n-2},
$$

and where we take the initial condition $\boldsymbol{u}_{-1}=\boldsymbol{u}_{0}=\boldsymbol{u}(0)$.

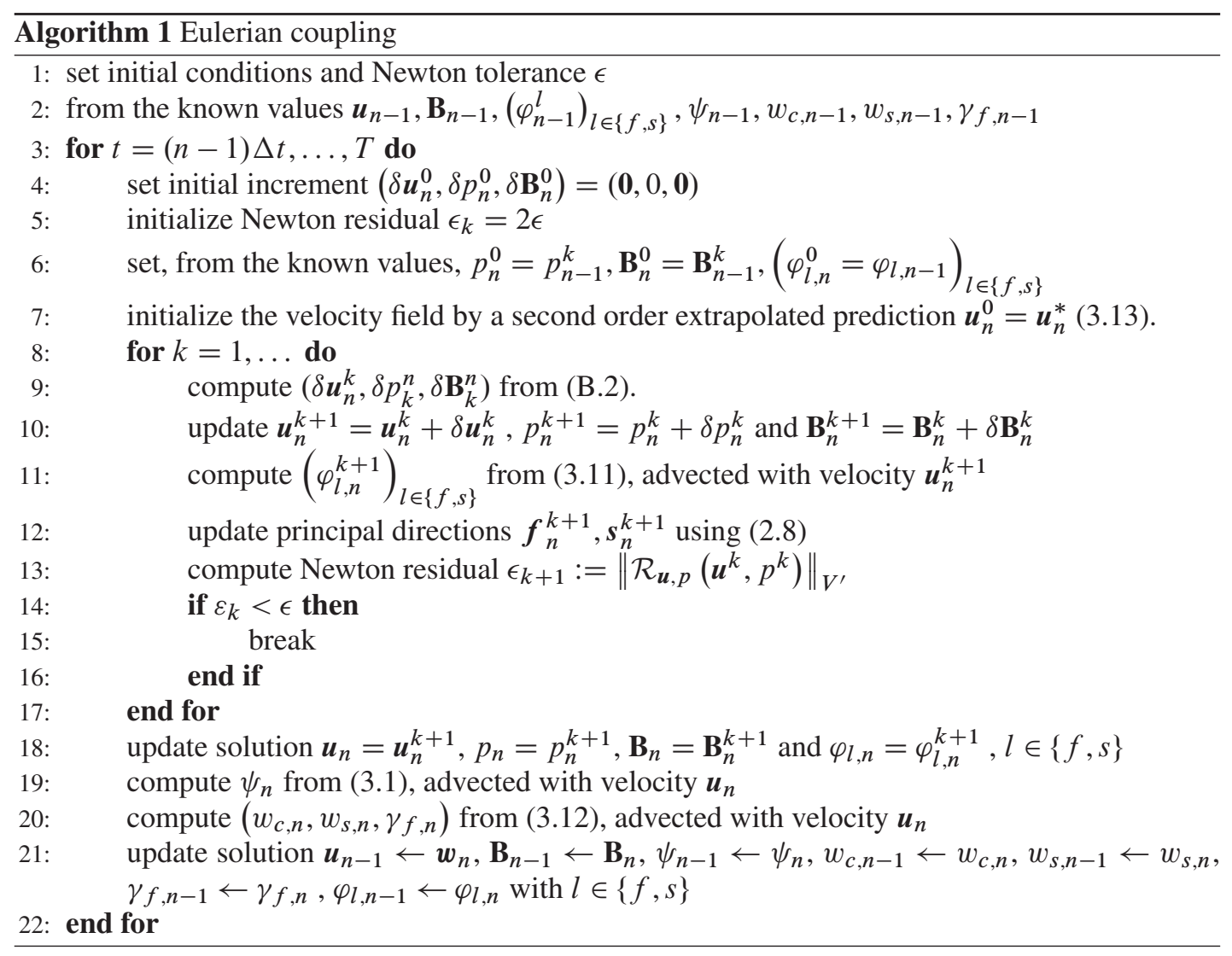




\subsection{Space discretization by finite elements}

We consider a partition $\mathscr{T}_{h}$ of $\Lambda$ consisting of geometrically conforming simplicial elements $K$ (tetrahedra for $d=3$, or triangles for $d=2$ ), such that $\bar{\Lambda}=\cup_{K \in \mathscr{T}_{h}}$. A Taylor-Hood finite element approximation (see, e.g., [40]) is considered for the discretization of velocity and pressure on a mesh of size $h=\max _{K \in \mathscr{T}_{h}} \operatorname{diam}(K)$. Let us denote by $\varphi_{n, h}^{k}$ an approximation of $\varphi_{n}^{k}$ at the $k$-th Newton iteration of time step $n$ and consider the following finite dimensional spaces:

$$
\begin{aligned}
& Q_{h}=\left\{q_{h} \in C^{0}(\bar{\Lambda}): q_{h \mid K} \in \mathbb{P}_{1}, \forall K \in \mathscr{T}_{h}\right\}, V_{h}=\left\{\boldsymbol{u}_{h} \in V \cap C^{0}(\bar{\Lambda})^{d}: \boldsymbol{u}_{h \mid K} \in \mathbb{P}_{2}^{d}, \forall K \in \mathscr{T}_{h}\right\}, \\
& M_{h}=\left\{\boldsymbol{\tau}_{h} \in M: \boldsymbol{\tau}_{h}=\boldsymbol{\tau}_{h}^{T}, \boldsymbol{\tau}_{h, i j \mid K} \in \mathbb{P}_{1}, \forall K \in \mathscr{T}_{h}, 1 \leqslant i, j \leqslant d\right\} .
\end{aligned}
$$

The Galerkin approximation of (B.2) consists in finding $\left(\delta \boldsymbol{u}_{n, h}^{k}, \delta p_{n, h}^{k}, \delta \mathbf{B}_{n, h}^{k}\right) \in V_{h} \times Q_{h} \times M_{h}$ such that

$$
\begin{aligned}
\left\langle\mathcal{A}\left(\delta \boldsymbol{u}_{n, h}^{k}\right), \boldsymbol{v}_{h}\right\rangle+\left\langle\mathcal{B}\left(\delta p_{n, h}^{k}\right), \boldsymbol{v}_{h}\right\rangle+ & \sum_{i \in I}\left\langle\mathcal{F}_{i} \mathcal{G}^{-1} \mathcal{H}_{i}\left(\delta \boldsymbol{u}_{n, h}^{k}\right), \boldsymbol{v}_{h}\right\rangle \\
+\left\langle\mathcal{F}_{i} \mathcal{G}^{-1} \mathcal{I}_{i}\left(\delta \boldsymbol{u}_{n, h}^{k}\right), \boldsymbol{v}_{h}\right\rangle & =-\left\langle\mathcal{R}_{\boldsymbol{u}, p}\left(\boldsymbol{u}_{n, h}^{k}, p_{n, h}^{k}\right), \boldsymbol{v}_{h}\right\rangle, \\
\left\langle q_{h}, \mathcal{B}^{\prime}\left(\delta \boldsymbol{u}_{n, h}^{k}\right)\right\rangle & =-\left\langle\mathcal{R}_{p}\left(\boldsymbol{u}_{n, h}^{k}\right), q_{h}\right\rangle, \\
\left\langle\mathcal{J}\left(\delta \mathbf{B}_{n, h}^{k}\right), \boldsymbol{\tau}_{h}\right\rangle+\left\langle\mathcal{K}\left(\delta \boldsymbol{u}_{n, h}^{k}\right), \boldsymbol{\tau}_{h}\right\rangle & =-\left\langle\mathcal{R}_{\boldsymbol{u}, \mathbf{B}}\left(\mathbf{B}_{n, h}^{k}\right), \boldsymbol{\tau}_{h}\right\rangle,
\end{aligned}
$$

for all $\left(\boldsymbol{v}_{h}, q_{h}, \boldsymbol{\tau}_{h}\right) \in V_{h} \times Q_{h} \times M_{h}$, where the bilinear forms are specified in Appendix B.

We notice that the weights $w, \hat{w}, \tilde{w}, w_{i}, \boldsymbol{w}, \mathbf{B}_{i}$ employed in $\mathcal{A}, \mathcal{B}, \mathcal{F}_{i}, \mathcal{H}_{i}, \mathcal{I}_{i}, \mathcal{J}$ and $\mathcal{K}$ are approximated by their $\mathbb{P}_{1}$ interpolants. The regularization parameter is chosen as $\varepsilon=3 \tilde{h}_{K}$, where $\tilde{h}_{K}$ is the average mesh-size near the element $K$ (Section 2.4). In practice, we evaluate $\tilde{h}_{K}$ using the mass lumping matrix $\hat{S}$ associated to the bilinear form $m\left(\varphi_{h}, \psi_{h}\right)=\int_{\Lambda} \varphi_{h} \psi_{h}$, for all $\varphi_{h}, \psi_{h} \in Q_{h}$. This leads to a diagonal matrix where the $i^{\text {th }}$ diagonal component represents the area of an elementary cell $C_{i}$ centered in the vertex $\mathbf{v}_{i}$. The cell contains one third of the adjacent element to the vertex $\mathbf{v}_{i}$ and it is defined by joining the barycenters $\mathbf{b}_{K}$ of the elements $K$ in the neighborhood of this vertex with the centers of each edge in $\mathscr{T}_{h}$ containing this vertex (Figure 2). Then the square root of the diagonal components of $\hat{S}$ approximate the local meshsize, which is particularly convenient when the mesh is non-regular.

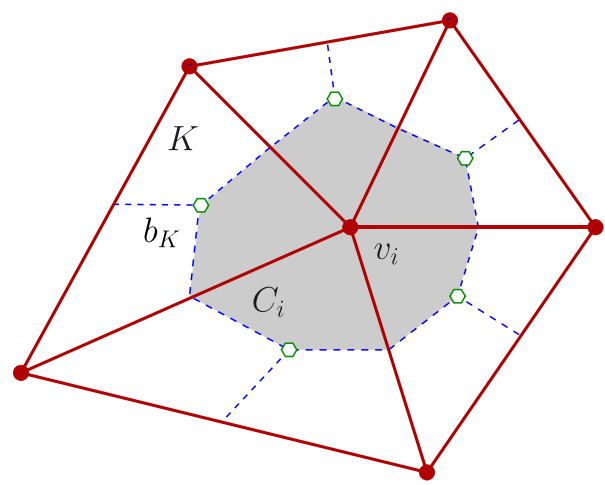

Figure 2. Elementary cell $C_{i}$ centered at the vertex $\mathbf{v}_{i}$. By $\mathbf{b}_{K}$, we denote the barycenter of $K \in \mathscr{T}_{h}$. 
(a)

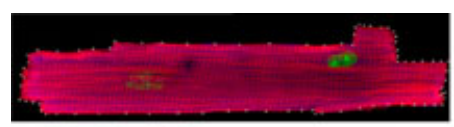

(d)

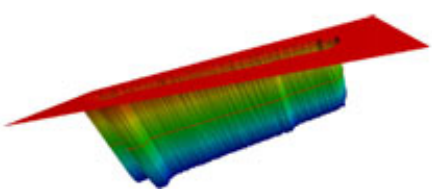

(b)

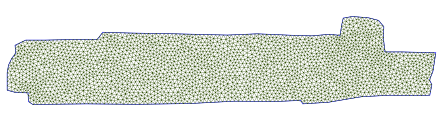

(e)

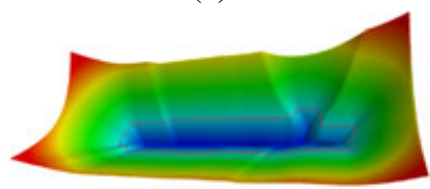

(c)

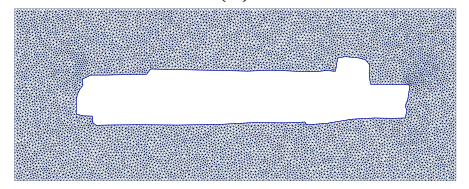

(f)

Figure 3. Level set initialization: (a) image from confocal microscopy [43] (cell size is $109 \mu m \times 26 \mu m$ ) and markers for the border identification, mesh for the (a) solid and (b) fluid domains, (d) initial interpolation,

(e) final level set profile, and (f) zero level sets before and after pre-processing (both curves coincide).

\subsection{Initialization of the level set function}

The generation of a signed distance field representation of the cell shape is needed to initialize the level set method. For simple topologies, an analytical description of $\Gamma$ is typically available, whereas in most cases, one only has access to images or to meshes with no fields defined on them. Here, a distance field is generated by computing the distance from every point to the discrete interface $\Gamma_{h}=\cup_{0 \leqslant i \leqslant N} E_{i}$ ( $E_{i}$ denotes an edge lying on the interface). For any node $\boldsymbol{x}_{0}$, we have

$$
\phi\left(x_{0}, \Gamma\right)=\min _{\boldsymbol{x} \in \Gamma_{h}}\left|x_{0}-\boldsymbol{x}\right|=\min _{0 \leqslant i \leqslant N}\left(\min _{\boldsymbol{x} \in E_{i}}\left|\boldsymbol{x}_{0}-\boldsymbol{x}\right|\right) .
$$

A sign may be associated to the obtained distance by direct computation in a separate loop over the mesh points [41] or by computing the normal vector to each element (see, e.g., [42]). These methods require a complete mesh scan to evaluate the signed distances. We here provide only negative and positive signs to vertexes inside and outside the solid, respectively, based on the resolution of the redistancing problem of Section 2.4.

Starting from an image of a cell obtained from confocal microscopy [43] (Figure 3(a)), the generation of the signed distance field requires a parametrization of the geometry using a given number of control points on the cell boundary, followed by a spline interpolation. An external box is created outside the body, on which a positive level set field is defined. We generate triangular meshes for $\Omega_{t}$ (Figure 3(b)), $\Gamma$ (set as internal boundary), and for $\Lambda / \Omega_{t}$ (Figure 3(c)). The whole pipeline is performed using the mesh manipulator Gmsh [44]. On the mesh, we define a scalar field $\phi_{0}$ that assumes the value 0 on $\Gamma,-1$ inside and +1 outside $\Omega_{t}$. However, $\phi_{0}$ has zero gradients inside and outside $\Gamma$ and therefore it is not appropriate to initialize (2.9). We then redefine $\phi_{0}$ as $f(\boldsymbol{x}) \times \phi_{0}(\boldsymbol{x})$, where $f(\boldsymbol{x}) \neq 0$ in $\Lambda$ (see Figure 3(d)) and we choose $f(\boldsymbol{x})=(x+10)(y+10)$ for $\Lambda=] 0,100[\times] 0,50[$. In addition, a mass lumping is used to smooth the initial level set behavior near $\Gamma$. That is, we solve $N$ times the matrix problem $\hat{S} \times \hat{\phi}_{0}=S \times \phi_{0}$, where $S, \widehat{S}$ denote the mass and mass lumping matrices. The value of the level set field in $\hat{\phi}_{0}\left(x_{0}\right)$ is then equal to the average value of $\phi_{0}$ over $N=3$ layers around $x_{0}$. The redistancing problem is then initialized with $\hat{\phi}_{0}$ until the steady state is reached and the solution is a signed distance and can therefore be employed as initial data for the coupled problem. A summary of the main steps of the pipeline is presented in Figure 3.

\section{NUMERICAL EXAMPLES}

All our numerical tests have been implemented using the open source finite element library Rheolef [45].

\subsection{Example 1: Solid motion in a lid-driven cavity flow}

We first study the motion of a deformable solid immersed in a lid-driven cavity following [46] (see also [47]). We perform a qualitative comparison with the results reported in [46], where the 
authors use a fixed-mesh finite difference method for the FSI problem and track the interface with a combination of immersed boundary and force-projection methods.

The deformation of the solid is governed by (2.6) with $b=a_{f}=a_{s}=a_{f s}=0$, and we consider different densities for the fluid and solid domains. The domain of interest is $\Lambda=] 0,1\left[{ }^{2}\right.$ and we assume that the system is initially at rest. The unstressed solid is a disk of radius 0.2 centered on $(0.6,0.5)$. At $t=0$, the top wall slides with a speed $\boldsymbol{w}=(1,0)$. The fluid viscosity is $\mu_{f}=0.01$, and we consider two cases with $a_{s}=0.01$ and $a_{s}=0.1$.
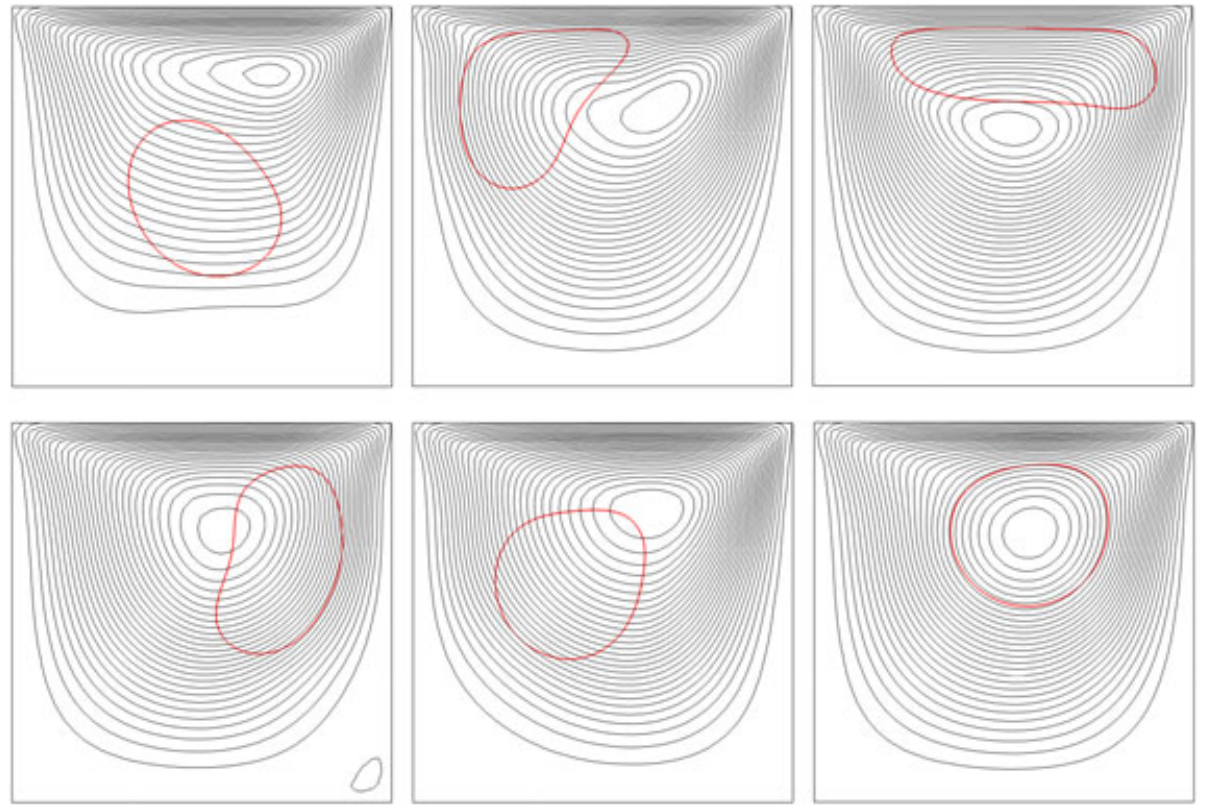

Figure 4. Example 1: Stream function contours for $R e=1, a_{s}=0.1$, and $\mu_{f}=0.01$, respectively, at times $t=1.25, t=4, t=5.5, t=7.25, t=9.5$, and $t=62$.
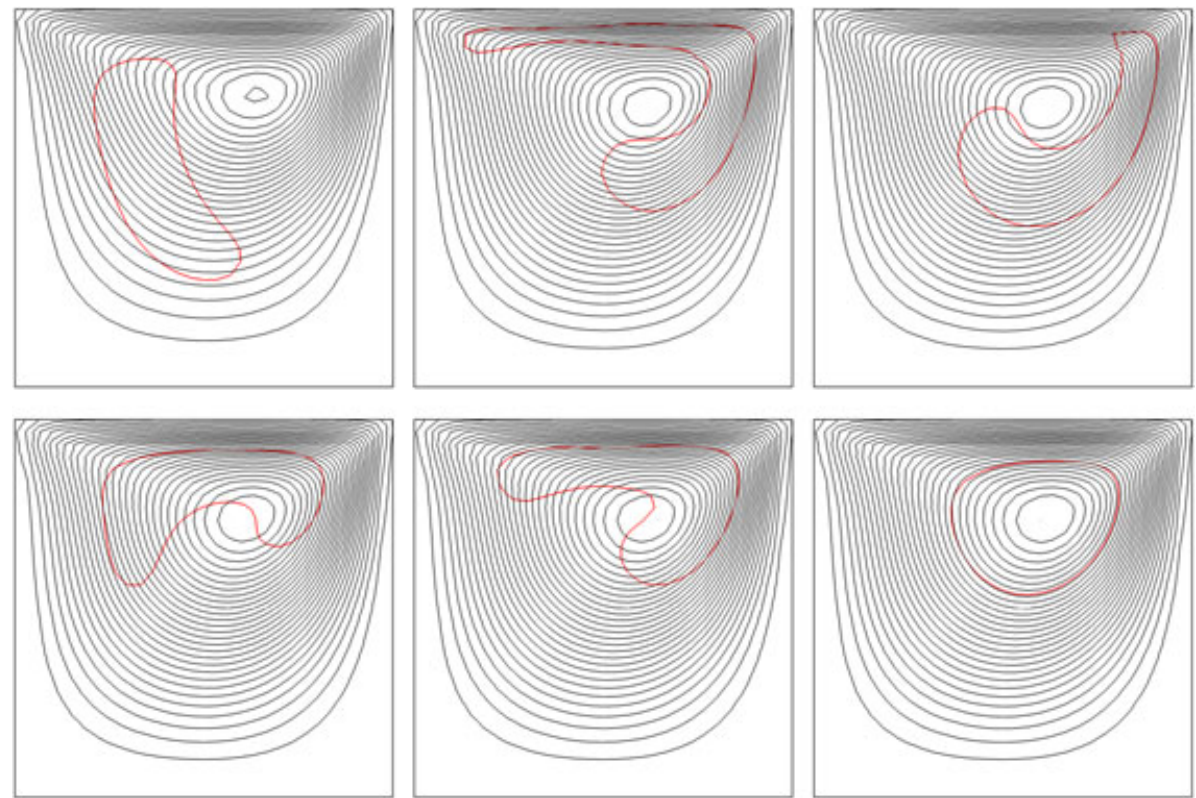

Figure 5. Example 1: Stream function contours for $R e=1$ and $\mu_{f}=a_{s}=0.01$, respectively, at times $t=1.25, t=3.25, t=3.75, t=5.25, t=5.75$, and $t=34.25$. 
In Figures 4 and 5, we visualize the particle deformation and the flow field for six consecutive snapshots. In both cases, we observe that the elastic body ends up near the center of gravity, and the flow field reaches a steady state. We also notice that the solid deformation is not symmetric around the disk's vertical centerline and the deformation is more evident in the case of smaller elastic modulus $a_{s}=0.1$, which is in agreement with respect to the results in [46]. To avoid contact between the solid and the top wall, we restrict the solid-wall distance to be larger than $\varepsilon=h$. In addition, we trace the centroid of the solid via the approximation $\boldsymbol{x}_{c}=\left(\int_{\Omega_{t}} \mathscr{H}_{\varepsilon}(\varphi) \boldsymbol{x} d \boldsymbol{x}\right)\left(\int_{\Omega_{t}} \mathscr{H}_{\varepsilon}(\varphi) d \boldsymbol{x}\right)^{-1}$.

Figure 6 shows the evolution of $\boldsymbol{x}_{c}$ for two different values of the elastic modulus, where a more regular trajectory is observed for $a_{s}=0.1$.
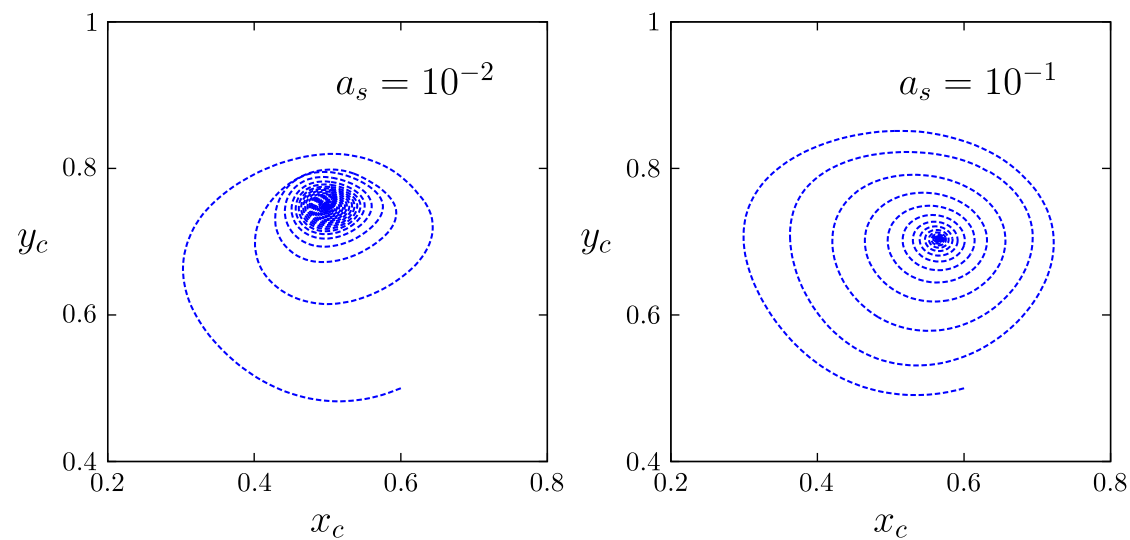

Figure 6. Example 1: Trajectories of the solid centroid over $t \in[0,150]$ for two values of $a_{s}$.

Table II. Example 2: Output values $\mathcal{J}_{i}$ for different values of $a_{s}$.

\begin{tabular}{|c|c|c|c|c|c|c|c|c|}
\hline$\mu_{f}$ & $a_{s}$ & $1 / h$ & $\mathcal{J}_{1}$ & $\mathcal{J}_{2}$ & $\mathcal{J}_{3}$ & $\mathcal{J}_{4}$ & $\mathcal{J}_{5}$ & $\mathcal{J}_{6}$ \\
\hline \multirow{7}{*}{0.01} & \multirow{7}{*}{0.05} & 10 & $-3.9624 \mathrm{E}-2$ & $2.1598 \mathrm{E}-2$ & $1.3130 \mathrm{E}-4$ & $2.1752 \mathrm{E}-1$ & $2.8589 \mathrm{E}-3$ & $9.7198 \mathrm{E}-3$ \\
\hline & & 16 & $-3.4698 \mathrm{E}-2$ & $2.1264 \mathrm{E}-2$ & $1.2911 \mathrm{E}-4$ & $1.3585 \mathrm{E}-1$ & $1.3137 \mathrm{E}-3$ & $3.9149 \mathrm{E}-3$ \\
\hline & & 25 & $-3.2272 \mathrm{E}-2$ & $1.9945 \mathrm{E}-2$ & $1.2354 \mathrm{E}-4$ & $8.6924 \mathrm{E}-2$ & $8.0848 \mathrm{E}-4$ & 4.4934E-3 \\
\hline & & 50 & $-2.9963 \mathrm{E}-2$ & $1.9361 \mathrm{E}-2$ & $1.1777 \mathrm{E}-4$ & $4.3458 \mathrm{E}-2$ & $4.7628 \mathrm{E}-4$ & $1.7027 \mathrm{E}-3$ \\
\hline & & 100 & $-2.8769 \mathrm{E}-2$ & $1.8999 \mathrm{E}-2$ & $1.1458 \mathrm{E}-4$ & $2.1730 \mathrm{E}-2$ & $2.7067 \mathrm{E}-4$ & $1.0052 \mathrm{E}-3$ \\
\hline & & 150 & $-2.8756 \mathrm{E}-2$ & $1.8940 \mathrm{E}-2$ & $1.1357 \mathrm{E}-4$ & $1.4489 \mathrm{E}-2$ & $2.2156 \mathrm{E}-4$ & $7.2550 \mathrm{E}-4$ \\
\hline & & 200 & $-2.8740 \mathrm{E}-2$ & $1.8911 \mathrm{E}-2$ & $1.1328 \mathrm{E}-4$ & $1.0867 \mathrm{E}-2$ & $1.7549 \mathrm{E}-4$ & $5.3330 \mathrm{E}-4$ \\
\hline \multirow{2}{*}{\multicolumn{3}{|c|}{$\begin{array}{l}\text { Reference values } \\
\text { Values from [15] }\end{array}$}} & $-2.8720 \mathrm{E}-2$ & $1.8771 \mathrm{E}-2$ & $1.1290 \mathrm{E}-4$ & - & - & - \\
\hline & & & $-2.8250 \mathrm{E}-2$ & $2.0550 \mathrm{E}-2$ & $1.6060 \mathrm{E}-4$ & - & - & - \\
\hline \multirow{7}{*}{0.01} & \multirow{7}{*}{0.10} & 10 & $-2.7814 \mathrm{E}-2$ & $1.2529 \mathrm{E}-2$ & $5.5647 \mathrm{E}-5$ & $2.1762 \mathrm{E}-1$ & $2.4577 \mathrm{E}-3$ & $3.7395 \mathrm{E}-3$ \\
\hline & & 16 & $-2.1338 \mathrm{E}-2$ & 1.1603E-2 & $4.7034 \mathrm{E}-5$ & $1.3589 \mathrm{E}-1$ & $1.1182 \mathrm{E}-3$ & $9.3158 \mathrm{E}-4$ \\
\hline & & 25 & $-2.0012 \mathrm{E}-2$ & $1.0766 \mathrm{E}-2$ & $4.0292 \mathrm{E}-5$ & $8.6937 \mathrm{E}-2$ & $7.1559 \mathrm{E}-4$ & $2.0117 \mathrm{E}-3$ \\
\hline & & 50 & $-1.6836 \mathrm{E}-2$ & $1.0125 \mathrm{E}-2$ & $3.6384 \mathrm{E}-5$ & $4.3464 \mathrm{E}-2$ & $4.1459 \mathrm{E}-4$ & $1.3799 \mathrm{E}-3$ \\
\hline & & 100 & $-1.6192 \mathrm{E}-2$ & $9.8720 \mathrm{E}-3$ & $3.4762 \mathrm{E}-5$ & $2.1732 \mathrm{E}-2$ & $2.1465 \mathrm{E}-4$ & $6.7208 \mathrm{E}-4$ \\
\hline & & 150 & $-1.5777 \mathrm{E}-2$ & $9.8210 \mathrm{E}-3$ & $3.4295 \mathrm{E}-5$ & $1.4490 \mathrm{E}-2$ & $1.8331 \mathrm{E}-4$ & $4.3927 \mathrm{E}-4$ \\
\hline & & 200 & $-1.5646 \mathrm{E}-2$ & $9.7830 \mathrm{E}-3$ & $3.4066 \mathrm{E}-5$ & $1.0868 \mathrm{E}-2$ & $1.3689 \mathrm{E}-4$ & $3.1633 \mathrm{E}-4$ \\
\hline \multirow{2}{*}{\multicolumn{3}{|c|}{$\begin{array}{l}\text { Reference values } \\
\text { Values from [15] }\end{array}$}} & $-1.4601 \mathrm{E}-2$ & $9.4760 \mathrm{E}-3$ & $3.2615 \mathrm{E}-5$ & - & - & - \\
\hline & & & $-1.4600 \mathrm{E}-2$ & $1.0080 \mathrm{E}-2$ & $3.9290 \mathrm{E}-5$ & - & - & - \\
\hline \multirow{7}{*}{0.01} & \multirow{7}{*}{1.00} & 10 & $-6.1388 \mathrm{E}-3$ & $1.8185 \mathrm{E}-3$ & $2.1538 \mathrm{E}-6$ & $2.1802 \mathrm{E}-1$ & $1.0249 \mathrm{E}-3$ & $1.5142 \mathrm{E}-5$ \\
\hline & & 16 & $-2.6605 \mathrm{E}-3$ & $1.3076 \mathrm{E}-3$ & $1.6636 \mathrm{E}-6$ & $1.3592 \mathrm{E}-1$ & $8.3094 \mathrm{E}-4$ & $9.7748 \mathrm{E}-6$ \\
\hline & & 25 & $-2.6123 \mathrm{E}-3$ & $1.2766 \mathrm{E}-3$ & $9.0292 \mathrm{E}-7$ & $8.6937 \mathrm{E}-2$ & $7.1559 \mathrm{E}-4$ & $7.0117 \mathrm{E}-6$ \\
\hline & & 50 & $-2.2240 \mathrm{E}-3$ & $1.1221 \mathrm{E}-3$ & $4.1097 \mathrm{E}-7$ & $4.3461 \mathrm{E}-2$ & $1.2846 \mathrm{E}-4$ & $4.4354 \mathrm{E}-6$ \\
\hline & & 100 & $-1.8130 \mathrm{E}-3$ & $1.0433 \mathrm{E}-3$ & $3.4370 \mathrm{E}-7$ & $2.1726 \mathrm{E}-2$ & $7.4604 \mathrm{E}-5$ & $4.1837 \mathrm{E}-6$ \\
\hline & & 150 & $-1.7516 \mathrm{E}-3$ & $1.0369 \mathrm{E}-3$ & $3.3874 \mathrm{E}-7$ & $1.4488 \mathrm{E}-2$ & $5.6782 \mathrm{E}-5$ & $3.0913 \mathrm{E}-6$ \\
\hline & & 200 & $-1.7434 \mathrm{E}-3$ & $1.0210 \mathrm{E}-3$ & $3.2703 \mathrm{E}-7$ & $1.0864 \mathrm{E}-2$ & $4.8826 \mathrm{E}-5$ & $3.0657 \mathrm{E}-6$ \\
\hline \multirow{2}{*}{\multicolumn{3}{|c|}{$\begin{array}{l}\text { Reference values } \\
\text { Values from [15] }\end{array}$}} & $-1.6773 \mathrm{E}-3$ & $8.9420 \mathrm{E}-4$ & $2.3331 \mathrm{E}-7$ & - & - & - \\
\hline & & & $-1.5120 \mathrm{E}-3$ & $9.8900 \mathrm{E}-4$ & $3.8920 \mathrm{E}-7$ & - & - & - \\
\hline
\end{tabular}




\subsection{Example 2: Driven cavity with elastic bottom}

We now focus on the steady-state behavior of a fluid immersed in a cavity with an elastic bottom. The domain $\Lambda=] 0,1\left[^{2}\right.$ is split into a structure and a fluid domain $\left.\Omega_{s}=\right] 0,1[\times] 0,1 / 4[$ and $\left.\Omega_{f}=\right] 0,1[\times] 1 / 4,1\left[\right.$, respectively. At the upper boundary of $\Omega_{f}$, the fluid undergoes a smooth tangential velocity $\boldsymbol{u}=(4 x(1-x), 0)^{T}$, and the structure is fixed at the bottom. Homogeneous Dirichlet data are prescribed on the remaining boundaries and the physical parameters are set to $\rho_{f}=\rho_{s}=1$, $\mu_{f}=0.01$ and $a_{s}=0.05$. Following [15], we observe the following outputs: structural displacement at point $\xi_{0}=(1 / 4,1 / 4), L^{2}$ norm of the displacements, structure and fluid incompressibility constraints, and relative volume error:

$\mathcal{J}_{1}=\boldsymbol{d}_{x}\left(\xi_{0}\right), \mathcal{J}_{2}=\boldsymbol{d}_{y}\left(\xi_{0}\right), \mathcal{J}_{3}=\|\boldsymbol{d}\|_{0, \Omega_{s}}^{2}, \mathcal{J}_{4}=\|\operatorname{div} \boldsymbol{u}\|_{0, \Omega_{f}}, \mathcal{J}_{5}=\|1-J\|_{0, \Omega_{s}}, \mathcal{J}_{6}=\frac{V-V_{i}}{V_{i}}$

Table II depicts the computed functionals $\mathcal{J}_{i}$ for successively refined meshes and different values for $a_{s}$, along with reference values from [15]. A satisfactory agreement is observed as $h$ decreases. In addition, linear rates of convergence are obtained for the functionals related to incompressibility $\mathcal{J}_{4}, \mathcal{J}_{5}, \mathcal{J}_{6}$ (Figure 7 (a)), as predicted also by [48].

A further comparison is performed with respect to the Eulerian and ALE-based computations from [15]. Relative errors for $\mathcal{J}_{1}, \mathcal{J}_{2}$, and $\mathcal{J}_{3}$ are displayed in Figure 7 (b), (c), and (d), respectively. The same convergence rate is observed for all methods.
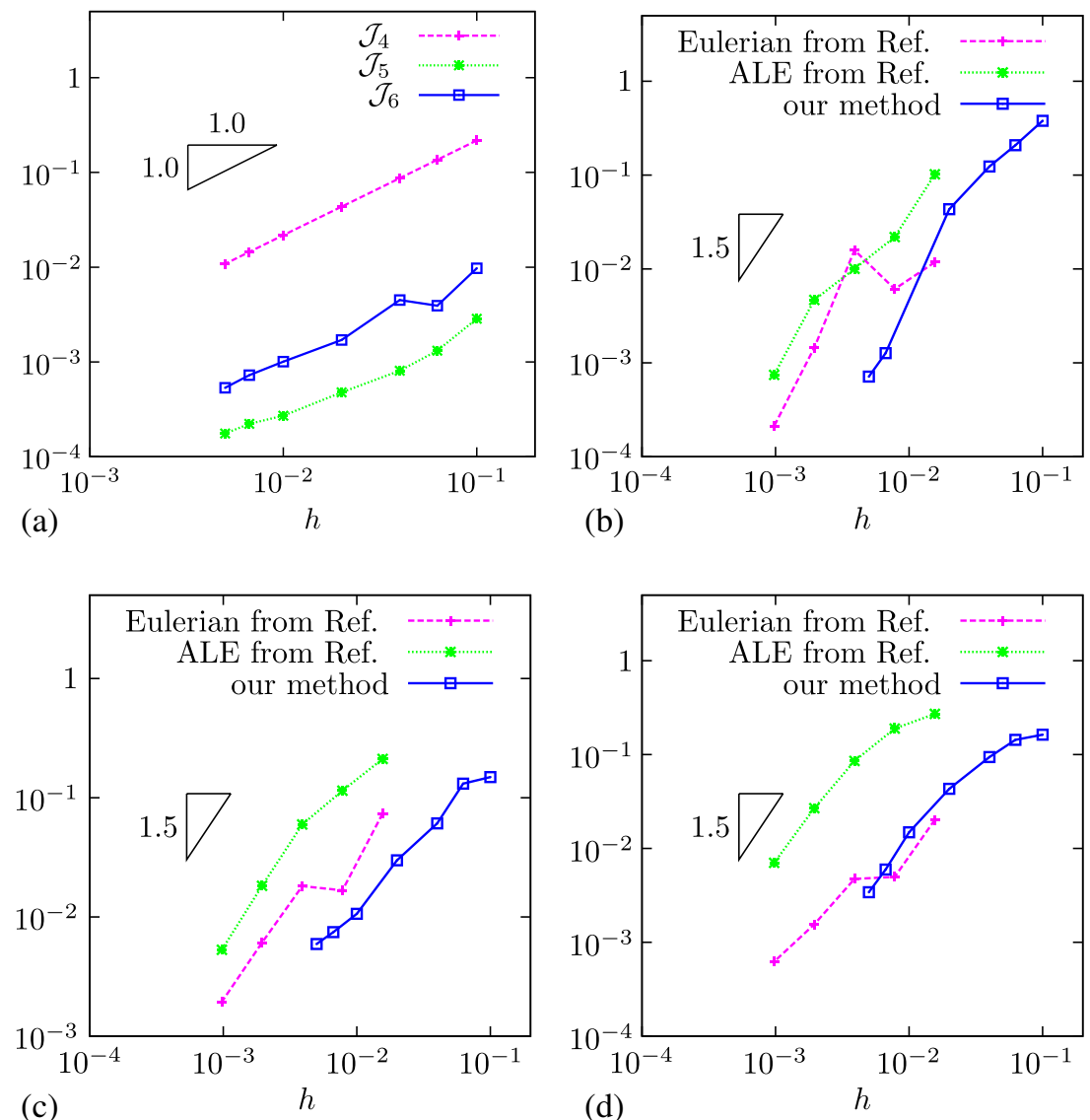

Figure 7. Example 2: Relative errors for the incompressibility-related functionals (a), and comparison with ALE and Eulerian methods from [15] in terms of errors for $\mathcal{J}_{1}(\mathrm{~b}), \mathcal{J}_{2}(\mathrm{c})$, and $\mathcal{J}_{3}(\mathrm{~d})$. 


\subsection{Example 3: Cardiomyocyte active contraction - grid convergence}

We now perform a fully Eulerian simulation of a deformable cardiomyocyte immersed in a fluid. The geometry has been segmented from a single adult rat cell morphology [43] (Section 3.5). The full anisotropic law is employed to describe the mechanical response of the cell. The spatial accuracy of the numerical approximations is studied by computing normalized errors in the energy norms on succesively refined meshes with respect to a highly accurate reference solution (obtained with a meshsize of $h=1 / 150$ ). These errors are computed at a final time $T=0.5 \mathrm{~s}$ and are defined as

$$
e(\boldsymbol{u}):=\frac{\left\|\boldsymbol{u}_{h}-\tilde{\boldsymbol{u}}\right\|_{\left(H^{1}(\Lambda)\right)^{d}}}{\|\tilde{\boldsymbol{u}}\|_{\left(H^{1}(\Lambda)\right)^{d}}}, \quad e(p):=\frac{\left\|p_{h}-\tilde{p}\right\|_{L^{2}(\Lambda)}}{\|\tilde{p}\|_{L^{2}(\Lambda)}}, \quad e(\mathbf{B}):=\frac{\left\|\mathbf{B}_{h}-\tilde{\mathbf{B}}\right\|_{\left(L^{2}(\Lambda)\right)^{d \times d}}}{\|\tilde{\mathbf{B}}\|_{\left(L^{2}(\Lambda)\right)^{d \times d}}},
$$

where the tildes refer to the refined reference solution. The error history is displayed in Figure 8 (top left panel), where we see suboptimal convergence rates, typically observed in multifluid models when the mesh is not fitted with the interface [35].

We now set the material parameters $b, a_{f}, a_{s}, a_{f s}$ to zero and study the evolution of the quantities

$$
\begin{aligned}
& \mathcal{L}_{1}=\frac{1}{T}\|\boldsymbol{u}\|_{0, \Omega_{t} ; T}, \mathcal{L}_{2}=\frac{1}{T}\left|\mathbf{B}_{11}\right|_{0, \Omega_{t} ; T}, \mathcal{L}_{3}=\frac{1}{T}\left|\mathbf{B}_{22}\right|_{0, \Omega_{t} ; T}, \mathcal{L}_{4}=\frac{1}{T}\left|I_{1}\right|_{0, \Omega_{t} ; T}, \mathcal{L}_{5}=\frac{1}{T}\left|I_{4, f}\right|_{0, \Omega_{t} ; T}, \\
& \mathcal{L}_{6}=\frac{1}{T}\left|\boldsymbol{\sigma}_{11}\right|_{0, \Omega_{t} ; T}, \mathcal{L}_{7}=\frac{1}{T}\left|\boldsymbol{\sigma}_{22}\right|_{0, \Omega_{t} ; T}, \mathcal{L}_{8}=\frac{1}{T}|\gamma|_{0, \Omega_{t} ; T}, \mathcal{L}_{9}=\frac{1}{T}\left|w_{c}\right|_{0, \Omega_{t} ; T}, \mathcal{L}_{10}=\frac{1}{T}\left|w_{s}\right|_{0, \Omega_{t} ; T},
\end{aligned}
$$
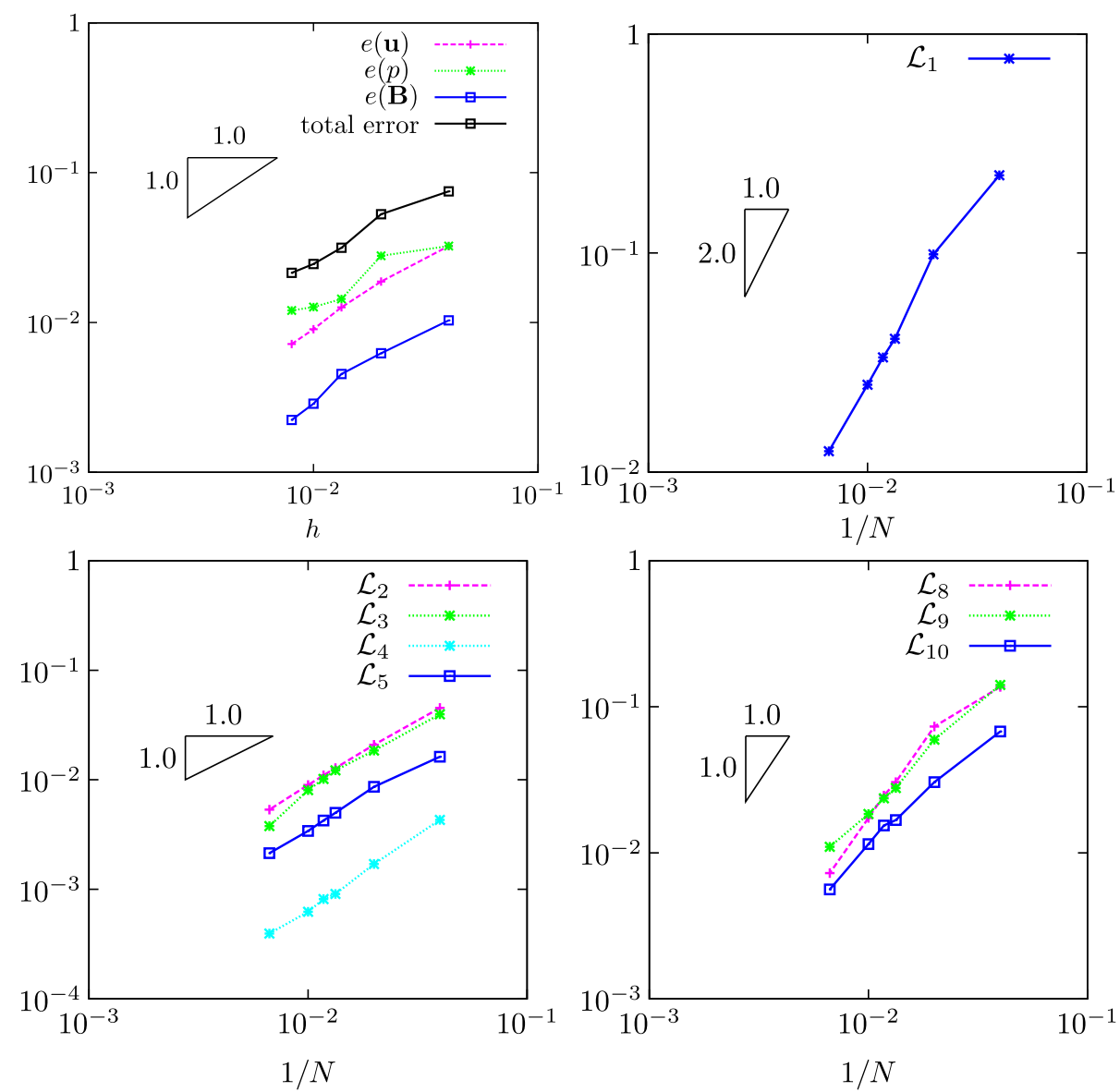

Figure 8. Example 3: Spatial convergence in the natural norms for a cell immersed in a fluid (top left), and convergence history of the outputs $\mathcal{L}_{i}$ with respect to spatial resolution. 


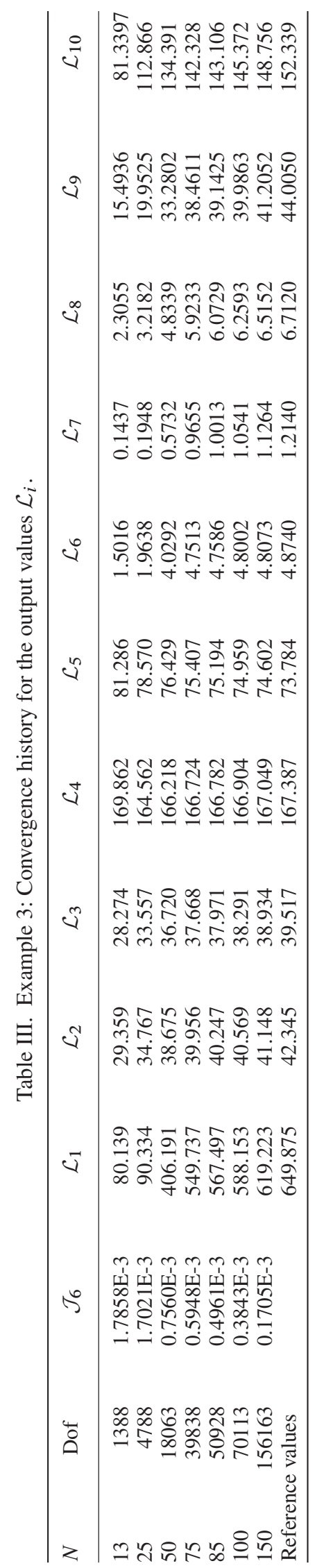


on the interval $t \in[0, T=8 s]$ for different meshsizes ( $N$ mesh points on the $x$-axis). Here, $\|\cdot\|_{0, \Omega_{t} ; T}$ and $|\cdot|_{0, \Omega_{t} ; T}$ denote the $L^{2}\left(0, T ;\left[L^{2}\left(\Omega_{t}\right)\right]^{2}\right)$ and $L^{2}\left(0, T ; L^{2}\left(\Omega_{t}\right)\right)$-norms, respectively. The remaining panels of Figure 8 show the mean $L^{2}$-norm as function of $N^{-1}$. An almost second-order accuracy for velocity and almost first-order accuracy for the components of $\mathbf{B}$ and the mechanical invariants is observed. The time step is small enough, and the time horizon is large enough to not influence substantially the overall accuracy. Table III reports the convergence of computed functionals along with reference values (obtained by numerical continuation). The time evolution of some of these quantities is shown in Figure 9, where a maximum stretch of about 20\% can be observed.

\subsection{Example 4: Cardiomyocyte active contraction - Validation of physiological conditions}

We now compare our results with those observed in [31]. Zero-stress boundary conditions are imposed on the fluid domain, and we study the local variation of the calcium wave front. In particular, we evaluate the propagation of the wavefront along the cell mean axis and compute the wavefront velocity. Figure 10 shows the trajectory and velocity of the calcium front for $t \in[0,1.1 \mathrm{~s}]$, and a satisfactory agreement is observed with respect to the published data.

We also study the cell shortening during contraction and compare with experimental data acquired from time-lapse contraction sequences using a combination of interface contrast, video-microscopy, and image correlation methods [49]. The calcium wave starts from the left side of the cell and we observe the time evolution of the average stretch $I_{4, f}$ (representing the rate of shortening with respect to the initial length in the direction of $\boldsymbol{f}_{0}$ ).

For these results, we have modified the kinetics of the activation, yielding to the specification $G\left(w_{c}, \gamma_{\mathrm{f}}\right)=-0.25[1 /(\mu \mathrm{Ms})] w_{c}-2.5[1 / s] \gamma_{\mathrm{f}}+0.09[1 / s]$. Initial conditions are set as $w_{c, 0}=$ $0.3586[\mu \mathrm{M}], w_{s, 0}=2.1436[\mu \mathrm{M}]$, and $\gamma_{f, 0}=-1.49 \mathrm{E}-4$, as seen in Figure 11(left).

From Figure 11 (middle), we observe a close fit of the simulated cell length with respect to the experiments. Displacements are obtained by integrating the first component of the intracellular strain
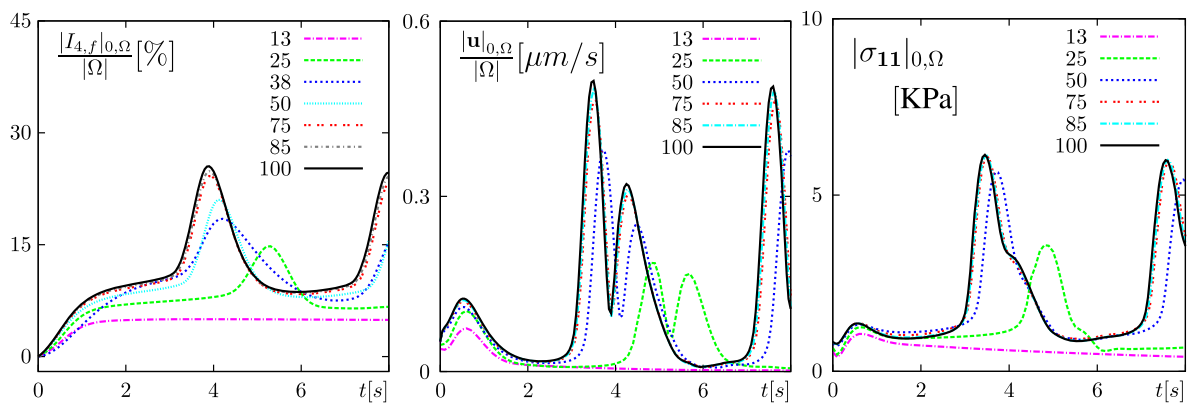

Figure 9. Example 3: Time evolution of some outputs for different spatial resolutions.
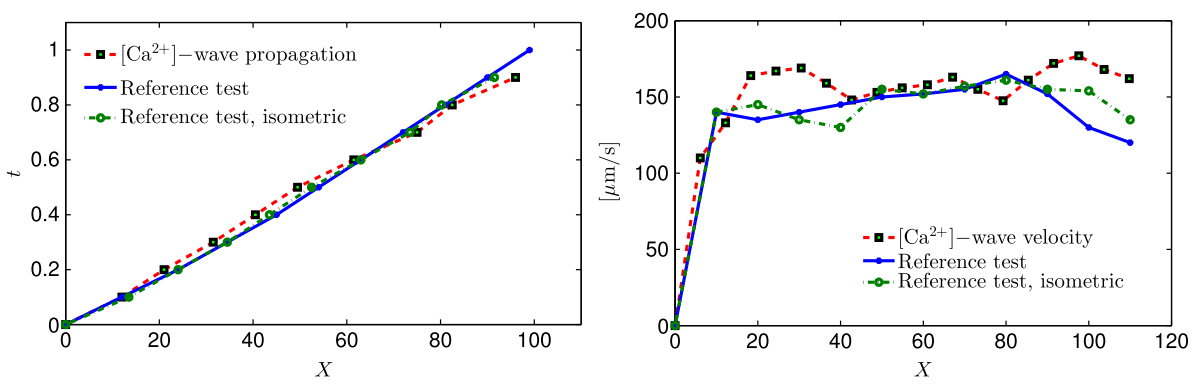

Figure 10. Example 4: Propagation of calcium (thick dashed red line) and comparison with unloaded (blue line) and isometric (cyan line) tests from [31] (left), and local variation of wave propagation velocity (thick red line) and comparison with tests for unloaded (blue line) and isometric (cyan line) tests (right). 

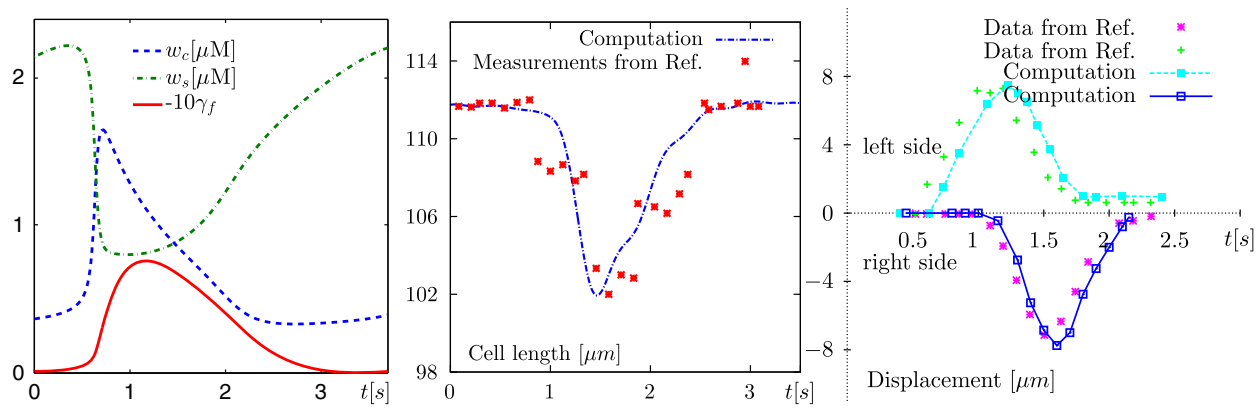

Figure 11. Example 4: Modified active strain behavior (left), and comparison with experiments from [49] in terms of cell length evolution (middle) and displacements on left and right ends of the myocyte (right).
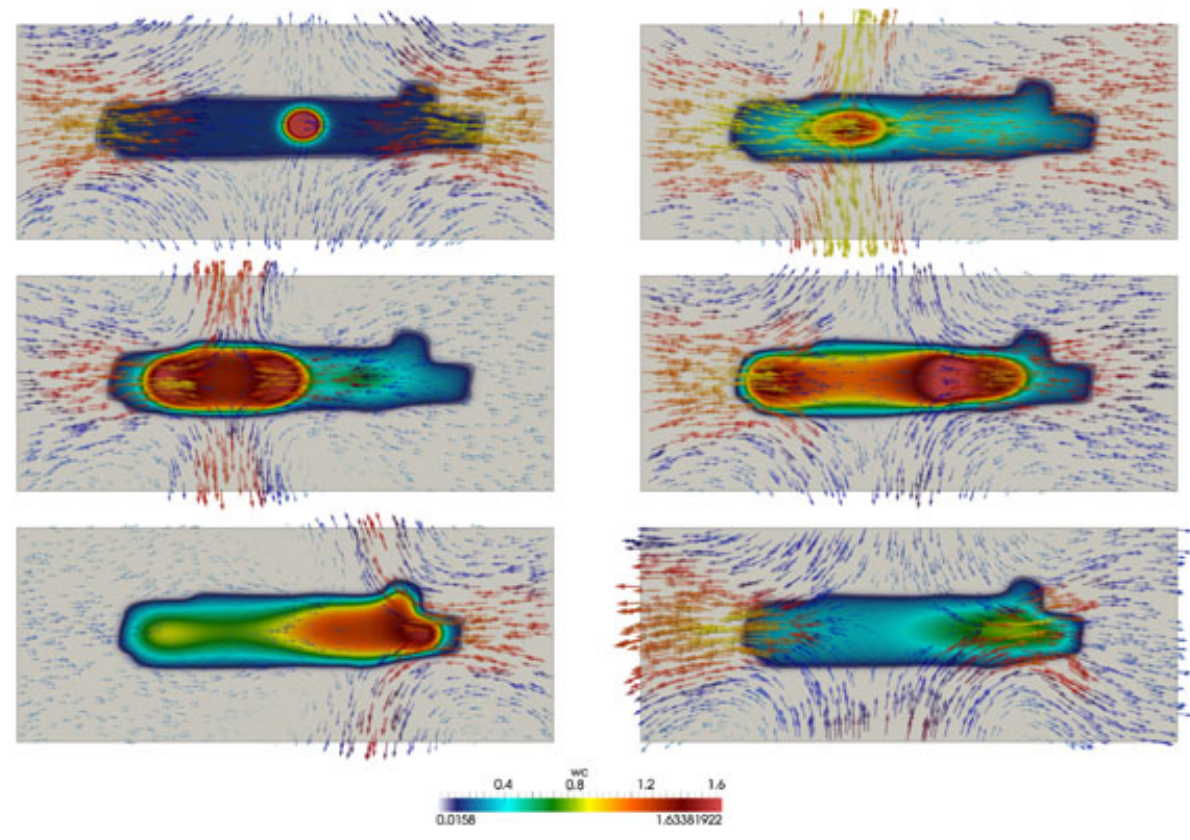

Figure 12. Example 5: Propagation of cytosolic calcium in the free cardiomyocyte and associated velocities for $t=0.05,3.35,3.5,3.75,4.3,8 \mathrm{~s}$ (from left to right and from top to bottom).

along the longitudinal cross-section at the middle of the cell. In both simulations and data, the fully contracted cell has a length of $102 \mu \mathrm{m}$, corresponding to a maximum contraction of about $10 \%$ with respect to the initial cell length. This takes place after $1.35 \mathrm{~s}$ from the start of the cycle. Details on the displacement of both extremities of the cardiomyocyte are presented in Figure 11 (right). Both experimental data and simulations show that the calcium wave propagation induces a phase shift in the displacement of the cell extremities. More precisely, the contraction of the right end is generated with a delay of about $0.37 \mathrm{~s}$ with respect to that on the left. We stress that these values were obtained with the aim to match the measured data from [49]. In experimental settings, the employed instruments are able to decrease (via pacing protocols) the intrinsic (much higher) frequency of the myocyte, to the values we recover herein.

\subsection{Example 5: Effect of boundary and initial conditions on contractile behavior}

In light of experimental observations from [34,50] and accordingly to the anisotropic diffusion of cytosolic calcium $w_{c}$, the orthonormal preferred directions of contraction have been initially set to $\boldsymbol{f}_{0}=(0,1)^{T}$ and $\boldsymbol{s}_{0}=(1,0)^{T}$, implying $\varphi_{0}^{f}(\boldsymbol{x})=x$ and $\varphi_{0}^{S}(\boldsymbol{x})=y$. Different contraction patterns have been simulated. First, (Figure 12) the cell has been let free to move and a localized cytosolic 
spark is originated from the center of the cell, which triggers two fronts of calcium waves that spread simultaneously towards the cell extremities. The cardiomyocyte is then stretched, and two intricate phases of contraction-relaxation interaction are visible on both ends. Such a contraction pattern in the longitudinal direction is associated to a relaxation in the transversal direction, also due to the incompressibility of the solid. Furthermore, one can see that calcium propagation induces cell bending, which is consistent with experimental observations reported in [51]. Figure 14 (bottom) depicts
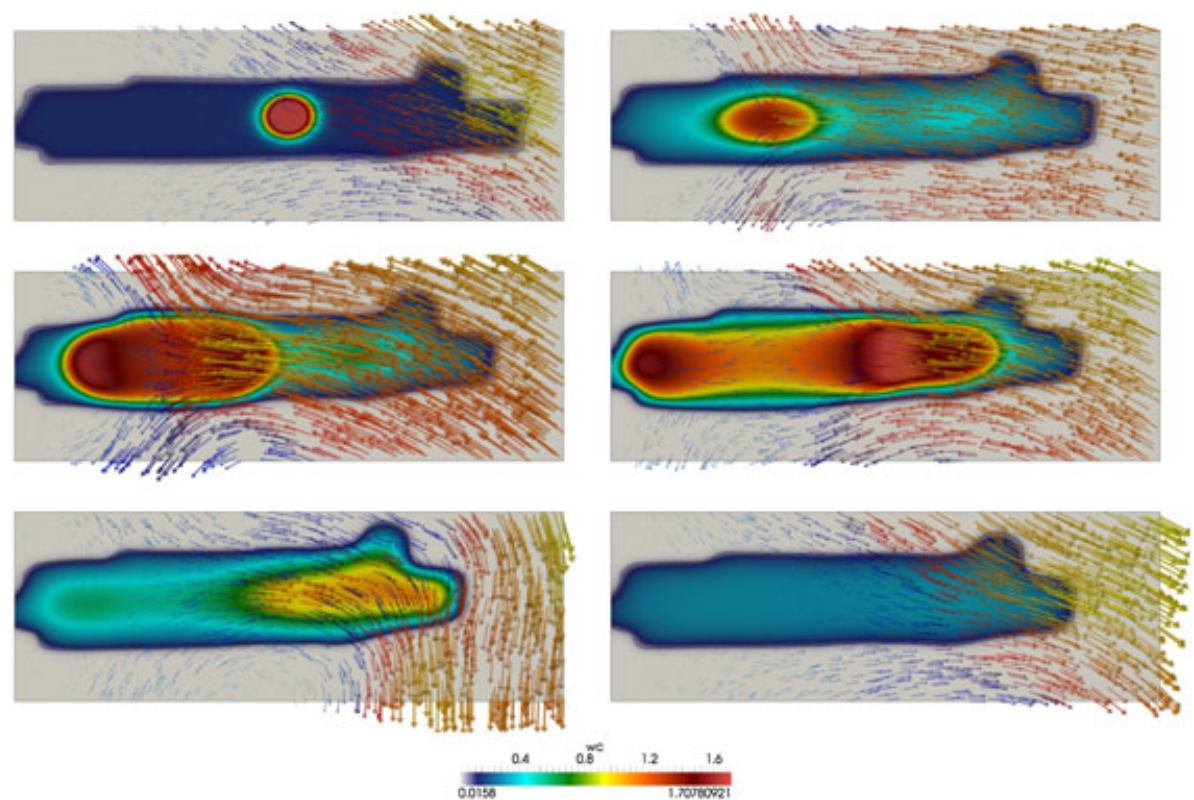

Figure 13. Example 5: Propagation of cytosolic calcium in the cell and associated velocities for $t=$ $0.05,3.25,3.55,3.65,4,6 \mathrm{~s}$ (from left to right and from top to bottom).
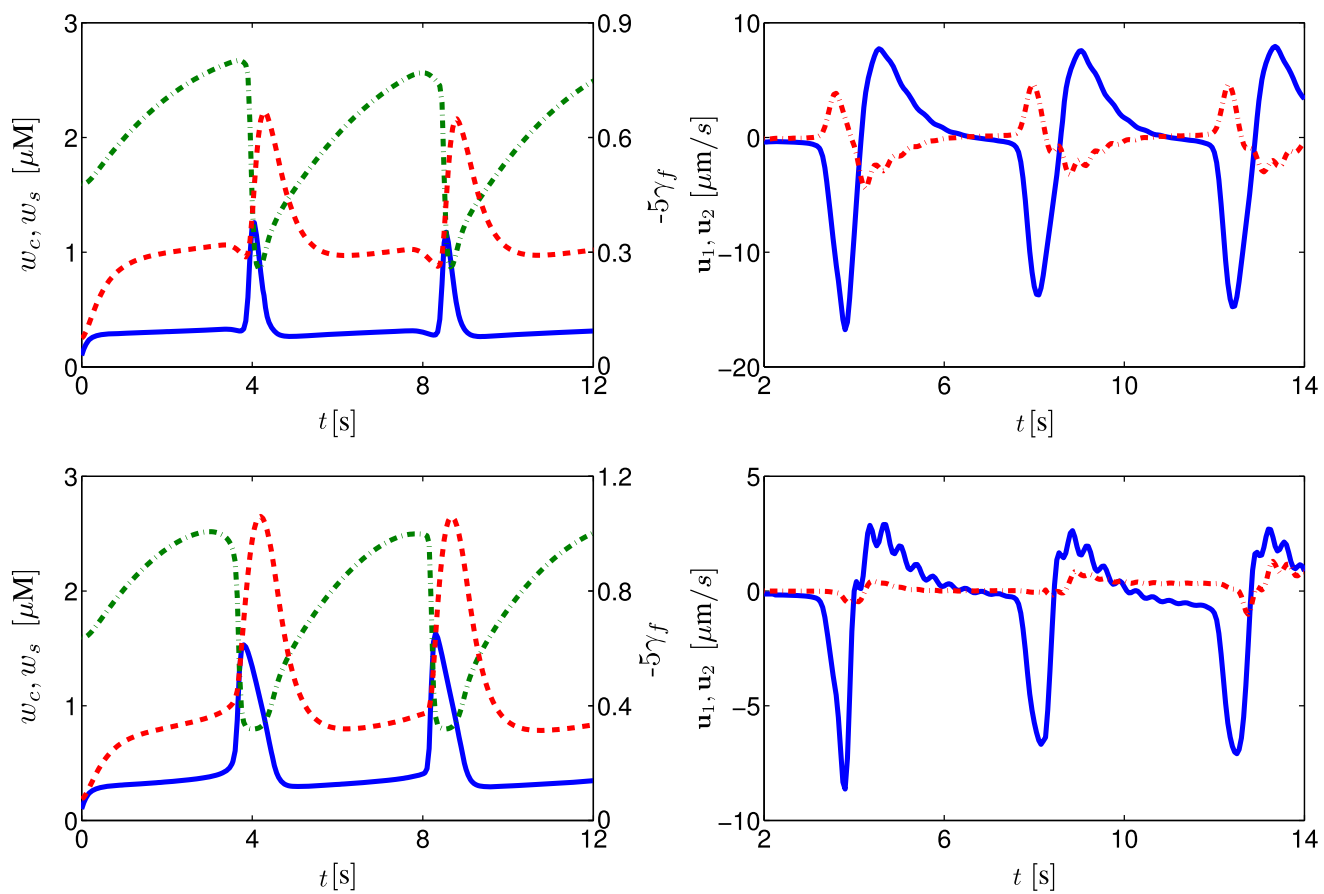

Figure 14. Example 5: Propagation of calcium concentrations and activation (left, in solid blue, dasheddotted green, and dashed red, respectively) and velocity components (right) on a single spatial point. 
the evolution of calcium, mechanical activation, and velocity components at a fixed point near the cell center. These results reveal that the transverse velocity has a small periodic pattern synchronized with the activation peak. The kinetics of $\left(w_{c}, w_{s}, \gamma_{\mathrm{f}}\right)$ agree with the predicted dynamics: a peak in cytosolic calcium triggers, up to a delay of about $0.2 \mathrm{~s}$, a peak in the activation, which induces a rhythmic contraction of the cardiomyocyte. Our simulations also indicate that the periodic pattern of contraction is significantly modified when the cell is no longer free to move in the fluid. After fixing the left end, one observes an increase of bending response (Figure 13). From Figure 14 (top) it is clear that calcium and activation patterns exhibit substantial differences with respect to the previous case. In particular, the bending is affected by the rhythmic patterns of the transversal velocity field.

\subsection{Example 6: Patterns of calcium propagation and activation}

A better understanding of the activation of the mechanical response is obtained by focusing on the spatio-temporal evolution of the intracellular calcium-driven activation. In Figure 15, we plot a line-scan showing successive snapshots of the propagation of intracellular calcium and activation waves along the cell main axis. This figure highlights the influence of the cytosolic calcium on triggering the cardiomyocyte contraction. From top to the bottom, one notices that a peak of cytosolic calcium induces two wave fronts propagating in opposite directions, generating simultaneously the activation maximum. Then, the activation reaches the cell extremities and generates maximum contractility before the degeneration of the wave front. These results are also in agreement with wavelengths of cytosolic calcium and active deformation patterns obtained with Lagrangian finite elements in [52]. As reported in [53], both contraction and intracellular calcium propagation patterns can be perturbed by particular dynamics near the cell nucleus. Even if these effects were not considered explicitly, the inclusion of different biomechanical properties on the nucleus or other organelles (as larger stiffness or heterogeneity in the conduction patterns) can be straightforwardly incorporated with a further level set function.
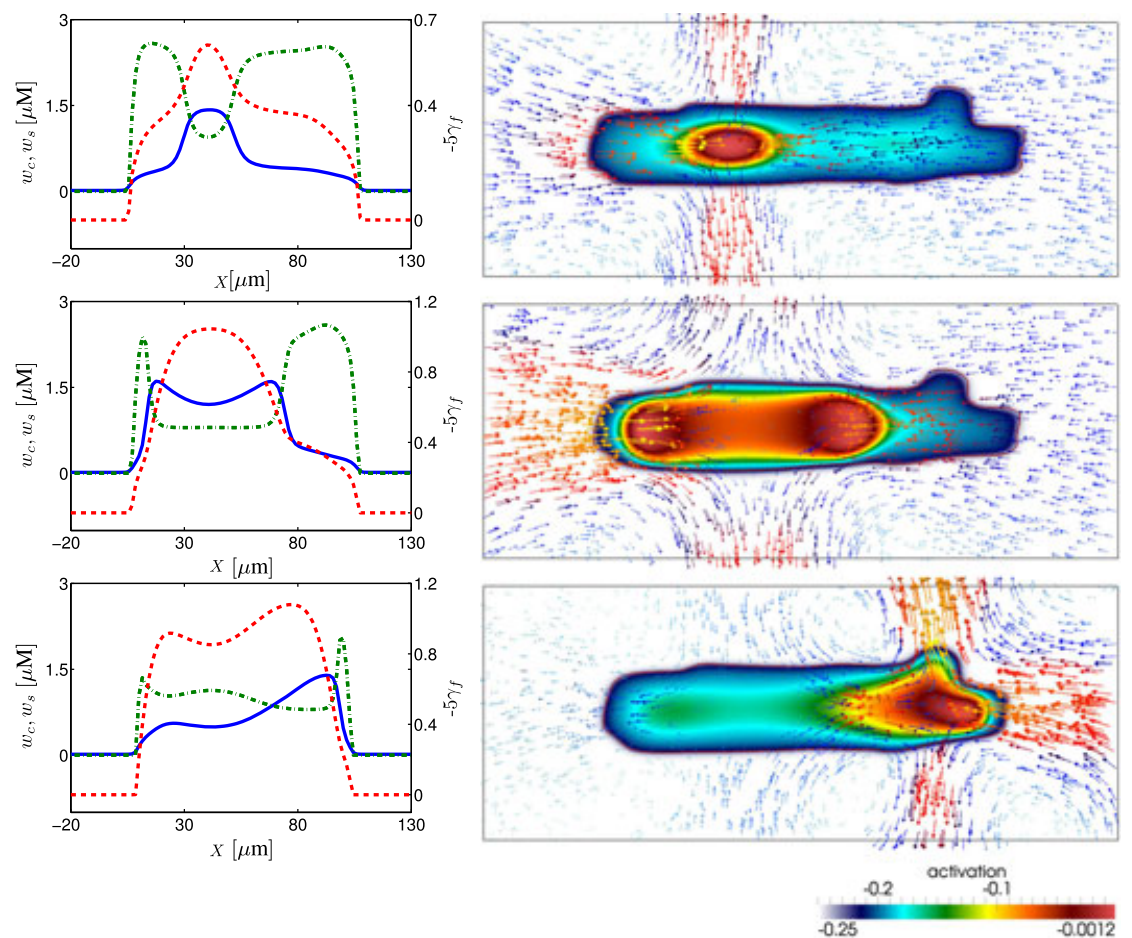

Figure 15. Example 6: Calcium and activation profiles (solid blue, dashed red, and dashed-dotted green, respectively) for $t=7.65,8,8.55 \mathrm{~s}$ (left panels), and activation patterns with velocity fields (right panels). 


\section{CONCLUDING REMARKS}

We have introduced an Eulerian finite element formulation for the numerical simulation of incompressible hyperelastic materials immersed in a Newtonian fluid and their interaction with chemical processes related to the release of cytosolic and sarcoplasmic calcium governed by reactiondiffusion equations. In summary, the main contributions of this work reside in that: (1) the mechanical description of the cell relies on a multiplicative decomposition of the deformation gradient into an elastic passive response and an anisotropic active component that is driven directly by the calcium release; (2) the Eulerian motion of the whole domain is characterized by transporting the left Cauchy-Green strain tensor rather than the inverse characteristics or deformations; (3) the fluidstructure interface is captured using a level set approach, which entails the use of a unified finite element solver for both fluid and solid. The cell mass conservation (crucial issue in Eulerian methods) is here enforced by a local Lagrange multiplier; (iv) a consistent Newton-Raphson linearization of the solid Cauchy stress tensor is applied, which guarantees stability for multiphysics coupling algorithms; (v) the numerical scheme is entirely based on finite elements; and (vi) several numerical examples are presented to address in detail the relevance of the mathematical model in terms of physiological meaning (propagation patterns and velocity of calcium waves, contractile response to mechanical activation) and the features of our numerical method in terms of spatio-temporal convergence and accuracy of the FSI coupling.

Some extensions of the developments in this paper are being currently explored. In particular, we are focusing on the construction of robust preconditioners that would allow substantial savings in computational effort. In addition, further simulations in a full three-dimensional setting are part of current developments, and we also foresee the applicability of the proposed framework to the simulation of macroscopic cardiac electromechanics (at both tissue and organ level).

\section{APPENDIX A: USEFUL DERIVATIVES AND LINEARIZATION EXPRESSIONS}

\section{Passive mechanics}

The derivatives of the invariants of the left Cauchy-Green deformation tensor $\mathbf{B}$ of (2.5) with respect to $\mathbf{F}$ are

$$
\frac{\partial I_{1}}{\partial \mathbf{F}}=2 \mathbf{F}, \quad\left(\frac{\partial I_{4, l}}{\partial \mathbf{F}}\right)_{l \in\{f, s\}}=2 \mathbf{F} \boldsymbol{l}_{0} \otimes \boldsymbol{l}_{0}, \quad \frac{\partial I_{8, f s}}{\partial \mathbf{F}}=\mathbf{F} \operatorname{sym}\left(\boldsymbol{f}_{0} \otimes \boldsymbol{s}_{0}\right) .
$$

For a given invariant $I_{j}$, we define $\psi_{j}:=\partial \mathcal{W} / \partial I_{j}$, where $\mathcal{W}$ is the internal stored energy function (2.4):

$\psi_{1}=\frac{a}{2} \exp \left(b\left[I_{1}-d\right]\right),\left(\psi_{4, l}\right)_{l \in\{f, s\}}=a_{l}\left(I_{4, l}-1\right) \exp \left(b_{l}\left[I_{4, l}-1\right]^{2}\right), \psi_{8, f_{s}}=a_{f_{s}} I_{8, f_{s}} \exp \left(b_{f_{s}} I_{8, f_{s}}^{2}\right)$.

Their derivatives with respect to $\mathbf{F}$ are

$$
\frac{\partial \psi_{1}}{\partial \mathbf{F}}=2 b \psi_{1} \mathbf{F}, \quad \frac{\partial \psi_{4, l}}{\partial \mathbf{F}}=\zeta_{4, l} \mathbf{F} \boldsymbol{l}_{0} \otimes \boldsymbol{l}_{0}, \text { with } l \in\{f, s\}, \quad \frac{\partial \psi_{8, f s}}{\partial \mathbf{F}}=\zeta_{8, f s} \mathbf{F} \operatorname{sym}\left(\boldsymbol{f}_{0} \otimes \boldsymbol{s}_{0}\right),
$$

where

$$
\zeta_{4, l}=2 a_{l}\left[2 b_{l}\left(I_{4, l}-1\right)^{2}+1\right] \exp \left(b_{l}\left[I_{4, l}-1\right]^{2}\right), \zeta_{8, f_{s}}=a_{f s}\left(2 b_{f_{s}} I_{8, f_{s}}^{2}+1\right) \exp \left(b_{f_{s}} I_{8, f_{s}}^{2}\right),
$$

with $l \in\{f, s\}$. The directional derivatives of some tensor quantities in the direction of a spatial velocity increment $\delta w$ are given by

$$
\begin{gathered}
D \mathbf{F}[\delta \boldsymbol{w}]=(\nabla \delta \boldsymbol{w}) \mathbf{F}, \quad D \mathbf{B}[\delta \boldsymbol{w}]=\operatorname{sym}(\nabla \delta \boldsymbol{w} \mathbf{B}), \quad D\left(\frac{\partial I_{1}}{\partial \mathbf{F}}\right)[\delta \boldsymbol{w}]=2 \nabla \delta \boldsymbol{w} \mathbf{F}, \\
D\left(\frac{\partial I_{4, l}}{\partial \mathbf{F}}\right)[\delta \boldsymbol{w}]=2 \nabla \delta \boldsymbol{w} \mathbf{F} \boldsymbol{l}_{0} \otimes \boldsymbol{l}_{0}, l=f, s, \quad D\left(\frac{\partial I_{8, f_{s}}}{\partial \mathbf{F}}\right)[\delta \boldsymbol{w}]=\nabla \delta \boldsymbol{w} \mathbf{F} \operatorname{sym}\left(\boldsymbol{f}_{0} \otimes \boldsymbol{s}_{0}\right),
\end{gathered}
$$


yielding the following expression for the linearization of the passive stress tensor $\mathbf{T}$ in the direction of $\delta \boldsymbol{u}$ :

$$
\begin{aligned}
D \mathbf{T}[\delta \boldsymbol{u}]= & \sum_{j}\left(\frac{\partial \psi_{j}}{\partial \mathbf{F}}: D \mathbf{F}[\delta \boldsymbol{u}]\right) \frac{\partial I_{j}}{\partial \mathbf{F}} \mathbf{F}^{T}+\sum_{j} \psi_{j}\left(\frac{\partial I_{j}}{\partial \mathbf{F}} D \mathbf{F}^{T}[\delta \boldsymbol{u}]+D\left(\frac{\partial I_{j}}{\partial \mathbf{F}}\right)[\delta \boldsymbol{u}] \mathbf{F}^{T}\right) \\
= & 4 b \psi_{1}(\mathbf{B}: \nabla \delta \boldsymbol{u}) \mathbf{B}+2 \psi_{1} \operatorname{sym}(\nabla \delta \boldsymbol{u} \mathbf{B}) \\
& +2 \zeta_{4, f}(\boldsymbol{f} \otimes \boldsymbol{f}: \nabla \delta \boldsymbol{u}) \boldsymbol{f} \otimes \boldsymbol{f}+4 \psi_{4, f} \operatorname{sym}(\nabla \delta \boldsymbol{u} \boldsymbol{f} \otimes \boldsymbol{f}) \\
& +2 \zeta_{4, s}(\boldsymbol{s} \otimes \boldsymbol{s}: \nabla \delta \boldsymbol{u}) \boldsymbol{s} \otimes \boldsymbol{s}+4 \psi_{4, s} \operatorname{sym}(\nabla \delta \boldsymbol{u} \boldsymbol{s} \otimes \boldsymbol{s}) \\
& +\zeta_{8, f s}(\operatorname{sym}(\boldsymbol{f} \otimes \boldsymbol{s}): \nabla \delta \boldsymbol{u}) \operatorname{sym}(\boldsymbol{f} \otimes \boldsymbol{s})+\psi_{8, f_{s}} \operatorname{sym}(\nabla \delta \boldsymbol{u} \operatorname{sym}(\boldsymbol{f} \otimes \boldsymbol{s})) .
\end{aligned}
$$

Active mechanics

Analogously, active scalar and tensor quantities along with their linearizations are defined as follows:

$$
\begin{aligned}
\frac{\partial I_{1}^{\mathrm{E}}}{\partial \mathbf{F}} & =2 \mathbf{F}-2 \sum_{l \in\{f, s, n\}} \frac{\gamma_{1}\left(\gamma_{1}+2\right)}{\left(\gamma_{1}+1\right)^{2}} \mathbf{F} \boldsymbol{l}_{0} \otimes \boldsymbol{l}_{0}, \frac{\partial I_{4, l}^{\mathrm{E}}}{\partial \mathbf{F}}=2\left(1+\gamma_{1}\right)^{-2} \mathbf{F} \boldsymbol{l}_{0} \otimes \boldsymbol{l}_{0}, \text { with } l \in\{f, s, n\}, \\
\frac{\partial I_{8, f s}^{\mathrm{E}}}{\partial \mathbf{F}} & =\left(1+\gamma_{\mathrm{f}}\right)^{-1}\left(1+\gamma_{\mathrm{s}}\right)^{-1} \mathbf{F} \operatorname{sym}\left(\boldsymbol{f}_{0} \otimes \beta_{0}\right) .
\end{aligned}
$$

The scalar coefficients $\psi_{j}^{\mathrm{E}}:=\partial \mathcal{W} / \partial I_{j}^{\mathrm{E}}$ are

$\psi_{1}^{\mathrm{E}}=\frac{a}{2} \exp \left(b\left[I_{1}^{\mathrm{E}}-d\right]\right), \psi_{4, l}^{\mathrm{E}}=a_{l}\left(I_{4, l}^{\mathrm{E}}-1\right) \exp \left(b_{l}\left[I_{4, l}^{\mathrm{E}}-1\right]^{2}\right), \psi_{8, f_{s}}^{\mathrm{E}}=a_{f_{s}} I_{8, f s}^{\mathrm{E}} \exp \left(b_{f_{s}}\left(I_{8, f s}^{\mathrm{E}}\right)^{2}\right)$,

and we have

$$
\begin{aligned}
& \frac{\partial \psi_{1}^{\mathrm{E}}}{\partial \mathbf{F}}=2 b \psi_{1}^{\mathrm{E}}\left(\mathbf{F}-\sum_{l \in\{f, s, n\}} \frac{\gamma_{1}\left(\gamma_{1}+2\right)}{\left(\gamma_{1}+1\right)^{2}} \mathbf{F} \boldsymbol{l}_{0} \otimes \boldsymbol{l}_{0}\right), \\
& \frac{\partial \psi_{4, l}^{\mathrm{E}}}{\partial \mathbf{F}}=\zeta_{4, l}^{E} \mathbf{F} \boldsymbol{l}_{0} \otimes \boldsymbol{l}_{0}, \quad \frac{\partial \psi_{8, f s}^{\mathrm{E}}}{\partial \mathbf{F}}=\zeta_{8, f s}^{E} \mathbf{F} \operatorname{sym}\left(\boldsymbol{f}_{0} \otimes \boldsymbol{s}_{0}\right),
\end{aligned}
$$

where

$$
\begin{aligned}
\zeta_{4, l}^{E} & =\frac{2 a_{l}}{\left(\gamma_{1}+1\right)^{2}}\left(2 b_{l}\left(I_{4, l}^{\mathrm{E}}-1\right)^{2}+1\right) \exp \left(b_{l}\left[I_{4, l}^{\mathrm{E}}-1\right]^{2}\right) \text { with } l \in\{f, s\}, \\
\zeta_{8, f s}^{E} & =\frac{a_{f s}}{\left(1+\gamma_{\mathrm{f}}\right)\left(1+\gamma_{\mathrm{s}}\right)}\left(2 b_{f s}\left(I_{8, f s}^{\mathrm{E}}\right)^{2}+1\right) \exp \left(b_{f_{s}}\left(I_{8, f s}^{\mathrm{E}}\right)^{2}\right) .
\end{aligned}
$$

The directional derivatives read

$$
\begin{aligned}
& D\left(\frac{\partial I_{1}^{\mathrm{E}}}{\partial \mathbf{F}}\right)[\delta \boldsymbol{u}]=2 \nabla \delta \boldsymbol{w} \mathbf{F}\left(\mathbf{I}-\sum_{l \in\{f, s, n\}} \frac{\gamma_{1}\left(\gamma_{1}+2\right)}{\left(\gamma_{1}+1\right)^{2}} \boldsymbol{l}_{0} \otimes \boldsymbol{l}_{0}\right) \\
& D\left(\frac{\partial I_{4, l}^{\mathrm{E}}}{\partial \mathbf{F}}\right)[\delta \boldsymbol{u}]=\frac{2}{\left(1+\gamma_{1}\right)^{2}} \nabla \delta \boldsymbol{w} \mathbf{F} \boldsymbol{l}_{0} \otimes \boldsymbol{l}_{0} ; l=f, s, D\left(\frac{\partial I_{8, f s}^{\mathrm{E}}}{\partial \mathbf{F}}\right)[\delta \boldsymbol{u}]=\frac{\nabla \delta \boldsymbol{w} \mathbf{F} \operatorname{sym}\left(\boldsymbol{f}_{0} \otimes \boldsymbol{s}_{0}\right)}{\left(1+\gamma_{\mathrm{f}}\right)\left(1+\gamma_{\mathrm{s}}\right)},
\end{aligned}
$$


which gives

$$
\begin{aligned}
D \mathbf{T}[\delta \boldsymbol{u}]= & \sum_{j}\left(\frac{\partial \psi_{j}^{\mathrm{E}}}{\partial \mathbf{F}}: D \mathbf{F}[\delta \boldsymbol{u}]\right) \frac{\partial I_{j}^{\mathrm{E}}}{\partial \mathbf{F}} \mathbf{F}^{T}+\sum_{j} \psi_{j}^{\mathrm{E}}\left(\frac{\partial I_{j}^{\mathrm{E}}}{\partial \mathbf{F}} D \mathbf{F}^{T}[\delta \boldsymbol{u}]+D\left(\frac{\partial I_{j}^{\mathrm{E}}}{\partial \mathbf{F}}\right)[\delta \boldsymbol{u}] \mathbf{F}^{T}\right) \\
= & 4 b \psi_{1}^{\mathrm{E}}\left(\hat{\mathbf{B}}_{1}: \nabla \delta \boldsymbol{u}\right) \hat{\mathbf{B}}_{1}+2 \psi_{1}^{\mathrm{E}} \operatorname{sym}\left(\nabla \delta \boldsymbol{u} \hat{\mathbf{B}}_{1}\right) \\
& +\zeta_{4, f}^{E}\left(\hat{\mathbf{B}}_{4, f}: \nabla \delta \boldsymbol{u}\right) \hat{\mathbf{B}}_{4, f}+\frac{2 \psi_{4, f}^{\mathrm{E}}}{\left(1+\gamma_{\mathrm{f}}\right)^{2}} \operatorname{sym}\left(\nabla \delta \boldsymbol{u} \hat{\mathbf{B}}_{4, f}\right) \\
& +\zeta_{4, s}^{E}\left(\hat{\mathbf{B}}_{4, s}: \nabla \delta \boldsymbol{u}\right) \hat{\mathbf{B}}_{4, s}+\frac{2 \psi_{4, s}^{\mathrm{E}}}{\left(1+\gamma_{\mathrm{s}}\right)^{2}} \operatorname{sym}\left(\nabla \delta \boldsymbol{u} \hat{\mathbf{B}}_{4, s}\right) \\
& +\zeta_{8, f s}^{E}\left(\hat{\mathbf{B}}_{8, f s}: \nabla \delta \boldsymbol{w}\right) \hat{\mathbf{B}}_{8, f s}+\frac{\psi_{8, f s}^{\mathrm{E}}}{\left(1+\gamma_{\mathrm{f}}\right)\left(1+\gamma_{\mathrm{s}}\right)} \operatorname{sym}\left(\nabla \delta \boldsymbol{w} \hat{\mathbf{B}}_{8, f s}\right),
\end{aligned}
$$

where $\hat{\mathbf{B}}_{1}=\mathbf{B}-\sum_{l \in\{f, s, n\}} \frac{\gamma_{1}\left(\gamma_{1}+2\right)}{\left(\gamma_{1}+1\right)^{2}} \boldsymbol{l} \otimes \boldsymbol{l}, \hat{\mathbf{B}}_{4, l}=\boldsymbol{l} \otimes \boldsymbol{l}, \hat{\mathbf{B}}_{8, f s}=\operatorname{sym}(\boldsymbol{f} \otimes \boldsymbol{s})$.

\section{APPENDIX B: ALTERNATIVE VARIATIONAL FORMULATION}

Let us define $\boldsymbol{\vartheta}_{i}^{k}=\left(\hat{\mathbf{B}}_{j}^{k}: \nabla \delta \boldsymbol{u}^{k}\right) \hat{\mathbf{B}}_{j}^{k}$ and $\boldsymbol{\zeta}_{j}^{k}=\operatorname{sym}\left(\nabla \delta \boldsymbol{u}^{k} \hat{\mathbf{B}}_{j}^{k}\right)$ with $j \in I$, and we denote

$$
\begin{aligned}
w_{1}^{k} & =4 b \psi_{1}^{\mathrm{E}, k} \mathscr{H}_{\varepsilon}\left(\psi_{n-1}\right), w_{i}^{k}=\zeta_{i}^{\mathrm{E}, k} \mathscr{H}_{\varepsilon}\left(\psi_{n-1}\right), z_{1}^{k}=2 \psi_{1}^{\mathrm{E}, k} \mathscr{H}_{\varepsilon}\left(\psi_{n-1}\right), \\
z_{4, f}^{k} & =\frac{2 \psi_{4, f}^{\mathrm{E}, k} \mathscr{H}_{\varepsilon}\left(\psi_{n-1}\right)}{\left(1+\gamma_{f, n-1}\right)^{2}}, z_{4, s}^{k}=\frac{2 \psi_{4, s}^{\mathrm{E}, k} \mathscr{H}_{\varepsilon}\left(\psi_{n-1}\right)}{\left(1+\gamma_{s, n-1}\right)^{2}}, z_{8, f s}^{k}=\frac{\psi_{8, f s}^{\mathrm{E}, k} \mathscr{H}_{\varepsilon}\left(\psi_{n-1}\right)}{\left(1+\gamma_{f, n-1}\right)\left(1+\gamma_{s, n-1}\right)},
\end{aligned}
$$

with $i \in\{(4, f),(4, s),(8, f s)\}$. In addition, we define the forms

$$
r(\boldsymbol{u}, \boldsymbol{\gamma} ; \mathbf{B})=\int_{\Lambda}(\mathbf{B}: \nabla \boldsymbol{u})(\mathbf{B}: \boldsymbol{\gamma}), \quad s(\boldsymbol{u}, \boldsymbol{\gamma} ; \mathbf{B})=\int_{\Lambda} \operatorname{sym}(\nabla \boldsymbol{u} \mathbf{B}): \boldsymbol{\gamma},
$$

for all $\boldsymbol{u} \in V, \boldsymbol{\gamma} \in M$, and $\mathbf{B} \in\left(L^{\infty}(\Lambda)\right)^{d \times d}$. Then, problem (3.10) reads: Find $\delta \boldsymbol{u}^{k}, \delta p^{k}, \delta \mathbf{B}^{k}, \vartheta_{i}^{k}, \boldsymbol{\zeta}_{i}^{k}$ such that

$$
\begin{aligned}
m\left(\delta \boldsymbol{u}^{k}, \boldsymbol{v} ; \frac{\rho_{\varepsilon}\left(\psi_{n-1}\right)}{\Delta t}\right)+c\left(\delta \boldsymbol{u}^{k}, \boldsymbol{v} ; \rho_{\varepsilon}\left(\psi_{n-1}\right), \boldsymbol{u}^{k}\right) & \\
+a\left(\delta \boldsymbol{u}^{k}, \boldsymbol{v} ; 1-\mathscr{H}_{\varepsilon}\left(\psi_{n-1}\right)\right)+b\left(\boldsymbol{v}, \delta p^{k}\right) & \\
+\sum_{i \in I} f\left(\boldsymbol{v}, \boldsymbol{\vartheta}_{i}^{k} ; w_{i}^{k}\right)+f\left(\boldsymbol{v}, \boldsymbol{\zeta}_{i}^{k} ; z_{i}^{k}\right) & =-\left\langle\mathcal{R}_{\boldsymbol{u}, p}\left(\boldsymbol{u}^{k}, p^{k}\right), \boldsymbol{v}\right\rangle_{V, V^{\prime}}, \\
g\left(\boldsymbol{\vartheta}_{i}^{k}, \boldsymbol{\gamma}\right) & =r\left(\delta \boldsymbol{u}^{k}, \boldsymbol{\gamma} ; \hat{\mathbf{B}}_{i}^{k}\right), \\
g\left(\boldsymbol{\zeta}_{i}^{k}, \boldsymbol{\gamma}\right) & =s\left(\delta \boldsymbol{u}^{k}, \boldsymbol{\gamma} ; \hat{\mathbf{B}}_{i}^{k}\right), \\
b\left(\delta \boldsymbol{u}^{k}, q\right) & =-\left\langle\mathcal{R}_{p}\left(\boldsymbol{u}^{k}\right), q\right\rangle_{Q, Q^{\prime}} \\
g\left(\frac{\delta \mathbf{B}^{k}}{\Delta t}, \boldsymbol{\tau}\right)+\alpha\left(\delta \mathbf{B}^{k}, \boldsymbol{\tau} ; \boldsymbol{u}^{k}\right)+\beta\left(\delta \boldsymbol{u}^{k}, \boldsymbol{\tau} ; \mathbf{B}^{k}\right) & =-\left\langle\mathcal{R}_{\boldsymbol{u}, \mathbf{B}}\left(\mathbf{B}^{k}\right), \boldsymbol{\tau}\right\rangle_{M, M^{\prime}},
\end{aligned}
$$


with $i \in I$, for all $(\boldsymbol{v}, q) \in V \times Q$ and $(\boldsymbol{\gamma}, \boldsymbol{\tau}) \in M$. Notice that the unknowns $\boldsymbol{\vartheta}_{i}^{k}, \boldsymbol{\varsigma}_{i}^{k}, i \in I$ can be eliminated by inverting the operator associated to the corresponding bilinear forms. In fact, for given regular weights $w, \hat{w}, \tilde{w}, w_{i}, \boldsymbol{w}, \mathbf{B}_{i}$ let us define the bounded weighted linear operators $\mathcal{A}: V \rightarrow V^{\prime}$, $\mathcal{B}: V \rightarrow Q^{\prime}, \mathcal{B}^{\prime}: Q \rightarrow V^{\prime}, \mathcal{F}_{i}: M \rightarrow V^{\prime}, \mathcal{G}: M \rightarrow M^{\prime},\left(\mathcal{H}_{i}, \mathcal{I}_{i}\right): V \rightarrow M^{\prime}, \mathcal{J}: M \rightarrow M^{\prime}$, $\mathcal{K}: V \rightarrow M^{\prime}$ as follows:

$$
\begin{aligned}
\langle\mathcal{A}(\boldsymbol{u}), \boldsymbol{v}\rangle & =m(\boldsymbol{u}, \boldsymbol{v} ; w)+c(\boldsymbol{u}, \boldsymbol{v} ; \hat{w}, \boldsymbol{u})+a(\boldsymbol{u}, \boldsymbol{v} ; \tilde{w}),\langle\mathcal{B}(\boldsymbol{v}), p\rangle=b(\boldsymbol{v}, \delta p), \\
\langle\mathcal{G}(\boldsymbol{\vartheta}), \boldsymbol{\tau}\rangle & =g(\boldsymbol{\vartheta}, \boldsymbol{\tau}),\left\langle\mathcal{F}_{i}(\boldsymbol{\vartheta}), \boldsymbol{v}\right\rangle=f\left(\boldsymbol{v}, \boldsymbol{\vartheta} ; w_{i}\right),\left\langle\mathcal{H}_{i}(\boldsymbol{u}), \boldsymbol{\tau}\right\rangle=r\left(\boldsymbol{u}, \boldsymbol{\tau} ; \mathbf{B}_{i}\right), \\
\left\langle\mathcal{I}_{i}(\boldsymbol{u}), \boldsymbol{\tau}\right\rangle & =s\left(\boldsymbol{u}, \boldsymbol{\tau} ; \boldsymbol{B}_{i}\right),\langle\mathcal{J}(\mathbf{B}), \boldsymbol{\tau}\rangle=\frac{1}{\Delta t} g(\mathbf{B}, \boldsymbol{\tau})+\alpha(\mathbf{B}, \boldsymbol{\tau} ; \boldsymbol{u}),\langle\mathcal{K}(\boldsymbol{u}), \boldsymbol{\tau}\rangle=\beta(\boldsymbol{u}, \boldsymbol{\tau} ; \mathbf{B}),
\end{aligned}
$$

for all $\boldsymbol{v} \in V, \boldsymbol{\tau} \in M$. Clearly, $\mathcal{G}^{-1}$ exists and we can write $\left\langle\boldsymbol{\vartheta}_{i}, \boldsymbol{\gamma}\right\rangle=\left\langle\mathcal{G}^{-1}\left(\mathcal{H}\left(\delta \boldsymbol{w}^{k}\right)\right), \boldsymbol{\gamma}\right\rangle$, for all $\gamma \in M$, which leads to

$$
\left\langle\mathcal{F}_{i}\left(\boldsymbol{\vartheta}_{i}\right), \boldsymbol{v}\right\rangle=\left\langle\mathcal{F}_{i}\left(\mathcal{G}^{-1}\left(\mathcal{H}_{i}\left(\delta \boldsymbol{u}^{k}\right)\right)\right), \boldsymbol{v}\right\rangle, \quad \forall \boldsymbol{v} \in V,
$$

and we proceed analogously for the equations corresponding to $\varsigma_{i}$ and $\mathcal{I}_{i}$. Then, system (B.1) reads: Find $\delta \boldsymbol{w}^{k}, \delta p^{k}$, and $\delta \mathbf{B}^{k}$ such that for all $(\boldsymbol{v}, q, \boldsymbol{\tau}) \in V \times Q \times M$,

$$
\begin{aligned}
\left\langle\mathcal{A}\left(\delta \boldsymbol{u}^{k}\right), \boldsymbol{v}\right\rangle+\left\langle\mathcal{B}\left(\delta p^{k}\right), \boldsymbol{v}\right\rangle+\sum_{i \in I}\left\langle\mathcal{F}_{i} \mathcal{G}^{-1} \mathcal{H}_{i}\left(\delta \boldsymbol{u}^{k}\right), \boldsymbol{v}\right\rangle+\left\langle\mathcal{F}_{i} \mathcal{G}^{-1} \mathcal{I}_{i}\left(\delta \boldsymbol{u}^{k}\right), \boldsymbol{v}\right\rangle & =-\left\langle\mathcal{R}_{\boldsymbol{w}, p}\left(\boldsymbol{u}^{k}, p^{k}\right), \boldsymbol{v}\right\rangle_{V, V^{\prime}}, \\
\left\langle q, \mathcal{B}^{\prime}\left(\delta \boldsymbol{u}^{k}\right)\right\rangle & =-\left\langle\mathcal{R}_{p}\left(\boldsymbol{u}^{k}\right), q\right\rangle_{Q^{\prime}, V} \\
\left\langle\mathcal{J}\left(\delta \mathbf{B}^{k}\right), \boldsymbol{\tau}\right\rangle+\left\langle\mathcal{K}\left(\delta \boldsymbol{u}^{k}\right), \boldsymbol{\tau}\right\rangle & =-\left\langle\mathcal{R}_{\boldsymbol{u}, \mathbf{B}}\left(\mathbf{B}^{k}\right), \boldsymbol{\tau}\right\rangle_{M, M^{\prime}}
\end{aligned}
$$

\section{ACKNOWLEDGEMENTS}

The authors thank Sathya Srinivasan for providing the cardiac cell confocal images and two anonymous referees whose suggestions resulted in a number of improvements of the paper. This work has been financially supported by the European Research Council through the Advanced Grant MATHCARD, Mathematical Modelling and Simulation of the Cardiovascular System, Project ERC-2008-AdG 227058.

\section{REFERENCES}

1. Sachse F. Computational Cardiology: Modeling of Anatomy, Electrophysiology, and Mechanics. Springer-Verlag: Berlin, 2004.

2. Kerckhoffs R, Healy S, Usyk T, McCulloch A. Computational methods for cardiac electromechanics. Proceedings of the IEEE 2006; 94:769-783.

3. Ruiz-Baier R, Ambrosi D, Pezzuto S, Rossi S, Quarteroni A. Activation models for the numerical simulation of cardiac electromechanical interactions. In Computer Models in Biomechanics: From Nano to Macro, Holzapfel G, Kuhl E (eds). Springer-Verlag: Heidelberg, 2013; 189-201.

4. Trayanova N. Whole-heart modeling: applications to cardiac electrophysiology and electromechanics. Circulation Research 2011; 108:113-128.

5. Lammerding J, Huang H, So P, Kamm R, Lee R. Quantitative measurements of active and passive mechanical properties of adult cardiac myocytes. IEEE Engineering in Medicine and Biology Magazine 2003; 22:124-127.

6. Weiwad W, Linke W, Wussling M. Sarcomere length-tension relationship of rat cardiac myocytes at lenghts greater than optimum. Journal of Molecular and Cellular Cardiology 2000; 32:247-259.

7. Iribe G, Helmes M, Kohl P. Force-length relations in isolated intact cardiomyocytes subjected to dynamic changes in mechanical load. American Journal of Physiology Heart and Circulatory Physiology 2007; 292:H1487-H1497.

8. Hirt C, Amsden A, Cook J. An arbitrary Lagrangian-Eulerian computing method for all flow speeds. Journal of Computational Physics 1997; 135(2):203-216.

9. Hughes TJ, Liu WK, Zimmermann TK. Lagrangian-Eulerian finite element formulation for incompressible viscous flows. Computer Methods in Applied Mechanics and Engineering 1981; 29(3):329-349.

10. Wick T. Fluid structure interactions using different mesh motion techniques. Computers \& Structures 2011; 89(13-14):1456-1467. 
11. Peskin C. The immersed boundary method. Acta Numerica 2002; 11:479-517.

12. Griffith B. On the volume conservation of the immersed boundary method. Communications in Computational Physics 2012; 12:401-432.

13. Dunne T. Adaptive finite element approximation of fluid-structure interaction based on eulerian and arbitrary Lagrangian-Eulerian variational formulations, Institute of Applied Mathematics, University of Heidelberg, 2007.

14. Rannacher R, Richter T. An adaptive finite element method for fluid-structure interaction problems based on a fully Eulerian formulation. In Fluid Structure Interaction II, Vol. 73, Bungartz H-J, Mehl M, Schfer M, Barth TJ, Griebel M, Keyes DE, Nieminen RM, Roose D, Schlick T (eds), Lecture Notes in Computational Science and Engineering. Springer: Berlin Heidelberg, 2010; 159-191.

15. Richter T, Wick T. Finite elements for fluid-structure interaction in ALE and fully Eulerian coordinates. Computer Methods in Applied Mechanics and Engineering 2010; 199:2633-2642.

16. Gueyffier D, Li J, Nadim A, Scardovelli R, Zaleski S. Volume-of-fluid interface tracking with smoothed surface stress methods for three-dimensional flows. Journal of Computational Physics 1999; 152:423-456.

17. Hirt C, Nichols B. Volume of fluid (vof) method for the dynamics of free boundaries. Journal of Computational Physics 1981; 39:201-225.

18. Allen S, Cahn J. A microscopic theory for antiphase boundary motion and its application to antiphase domain coarsening. Acta Materialia 1979; 27:1085-1095.

19. Du Q, Liu C, Ryham R, Wang X. Energetic variational approaches in modeling vesicle and fluid interactions. Physica D 2009; 238:923-930.

20. Okazawa S, Kashiyama K, Kaneko Y. Eulerian formulation using stabilized finite element method for large deformation solid dynamics. International Journal for Numerical Methods in Engineering 2007; 72:1544-1559.

21. Osher S, Sethian JA. Front propaging with curvature-dependent speed: agorithms based on Hamilton-Jacobi formulations. Journal of Computational Physics 1988; 79(1):12-49.

22. Cottet GH, Maitre E, Milcent T. Eulerian formulation and level set models for incompressible fluid-structure interaction. ESAIM: Mathematical Modelling and Numerical Analysis 2008; 42:471-492.

23. Maitre E, Milcent T, Cottet GH, Raoult A, Usson Y. Applications of level set methods in computational biophysics. Mathematical and Computer Modelling 2009; 49:2161-2169.

24. Laadhari A, Misbah C, Saramito P. On the equilibrium equation for a generalized biological membrane energy by using a shape optimization approach. Physica D 2010; 239:1567-1572.

25. Laadhari A, Saramito P, Misbah C. Computing the dynamics of biomembranes by combining conservative level set and adaptive finite element methods. Journal of Computational Physics, in press. Preprint available from http://hal.archives-ouvertes.fr/hal-00604145.

26. Nardinocchi P, Teresi L. On the active response of soft living tissues. Journal of Elasticity 2007; 88(1):27-39.

27. Rossi S, Ruiz-Baier R, Pavarino L, Quarteroni A. Orthotropic active strain models for the numerical simulation of cardiac biomechanics. International Journal for Numerical Methods in Biomedical Engineering 2012; 28 : 761-788

28. Nobile F, Quarteroni A, Ruiz-Baier R. An active strain electromechanical model for cardiac tissue. International Journal for Numerical Methods in Biomedical Engineering 2012; 28:52-71.

29. Miller G, Colella P. A high-order Eulerian Godunov method for elastic/plastic flow in solids. Journal of Computational Physics 2001; 167:131-176.

30. Holzapfel G, Ogden R. Constitutive modelling of passive myocardium: a structurally based framework for material characterization. Philosophical Transactions of the Royal Society London A 2009; 367:3445-3475.

31. Okada J, Sugiura S, Nishimura S, Hisada T. Three-dimensional simulation of calcium waves and contraction in cardiomyocytes using the finite element method. American Journal of Physiology - Cell Physiology 2005; 288:C510-C522.

32. Pustoc'h A, Boudou T, Ohayon J, Usson Y, Tracqui P. Finite element modelling of the calcium-induced contraction cardiomyocytes based on timelapse videomicroscopy. WSEAS Transactions on Information Science and Applications 2004; 1(1):376-378.

33. ter Keurs H. Electromechanical coupling in the cardiac myocyte; stretch-arrhythmia feedback. Pflügers Archiv European Journal of Physiology 2011; 462:165-175.

34. Tracqui P, Ohayon J. An integrated formulation of anisotropic force-calcium relations driving spatio-temporal contractions of cardiac myocytes. Philosophical Transactions of the Royal Society London A 2009; 367: 4887-4905.

35. Parolini N, Burman E. A finite element level set method for viscous free-surface flows. In Applied and Industrial Mathematics in Italy, Primicerio M, Spigler R, Valente V (eds). World Scientific: Singapore, 2005; 416-427.

36. Sussman M, Fatemi E, Smereka P, Osher S. An improved level set method for incompressible two-phase flows. Computers \& Fluids 1998; 27(5-6):663-680.

37. Gaudlitz D, Adams NA. On improving mass-conservation properties of the hybrid particle-level-set method. Computers \& Fluids 2008; 37(10):1320-1331.

38. Laadhari A, Saramito P, Misbah C. Improving the mass conservation of the level set method in a finite element context. Comptes Rendus Mathématiques Series I 2010; 348:535-540.

39. Pironneau O. On the transport-diffusion algorithm and its applications to the Navier-Stokes equations. Numerical Mathematics 1982; 38(3):309-332.

40. Brezzi F, Fortin M. Mixed and Hybrid Finite Element Methods. Springer Verlag: New York, 1991. 
41. Payne BA, Toga AW. Distance field manipulation of surface models. IEEE Computer Graphics and Applications 1992; 12(1):65-71.

42. Huang J, Li Y, Crawfis R, Lu SC, Liou SY. A complete distance field representation. Proceedings of the IEEE Visualization Conference 2001, Vol. 1, San Diego, USA, 2001; 247-254.

43. Srinivasan S. A method to fix and permeabilize isolated adult mouse cardiomyocytes for immuno-staining and confocal imaging. Nature Protocol Exchange 2011; 235. DOI: 10.1038/protex.2011.235.

44. Geuzaine C, Remacle JF. Gmsh: a three-dimensional finite element mesh generator with built-in pre- and post-processing facilities. International Journal for Numerical Methods in Engineering 2009; 79(11):1309-1331.

45. Saramito P. Efficient C++ finite element computing with Rheolef, CNRS and LJK, 2011. (Available from: http: //www-ljk.imag.fr/membres/Pierre.Saramito/rheolef) [Accessed on July 2012].

46. Zhao H, Freund J, Moser R. A fixed-mesh method for incompressible flow-structure systems with finite solid deformation. Journal of Computational Physics 2008; 227:3114-3140.

47. Gupta M, Kalita J. A new paradigm for solving Navier-Stokes equations: streamfunction-velocity formulations. Journal of Computational Physics 2005; 207:52-68.

48. Logg A, Mardal KA, Wells G. Automated Solution of Differential Equations by the Finite Element Method, Lecture Notes in Computational Science and Engineering, Vol. 84. Spinger-Verlag: Berlin Heidelberg, 2012.

49. Kamgoué A, Ohayon J, Usson Y, Riou L, Tracqui P. Quantification of cardiomyocyte contraction based on image correlation analysis. Cytometry Part A 2009; 75A(4):298-308.

50. Bourdarias C, Gerbi S, Ohayon J. A three dimensional finite element method for biological active soft tissueformulation in cylindrical polar coordinates. ESAIM: Mathematical Modelling and Numerical Analysis 2003; 37:725-739.

51. Delbridge L, Roos K. Optical methods to evaluate the contractile function of unloaded isolated cardiac myocytes. Journal of Molecular and Cellular Cardiology 1997; 29:11-25.

52. Ruiz-Baier R, Gizzi A, Rossi S, Cherubini C, Laadhari A, Filippi S, Quarteroni A. Mathematical modelling of active contraction in isolated cardiomyocytes. Mathematical Medicine and Biology 2013. In press.

53. Alonso M, Villalobos C, Chamero P, Alvarez J, García-Sancho J. Calcium microdomains in mitochondria and nucleus. Cell Calcium 2006; 40:513-525. 